\title{
Cylindrical Symmetry Discrimination of Magnetoelectric Optical Systematic Effects in a Pump-probe Atomic Parity Violation Experiment
}

\author{
M-A. Bouchiat, J. Guéna, M. Lintz \\ Laboratoire Kastler Brossel* et Fédération de Recherche ${ }^{\dagger}$ \\ Département de Physique de l'Ecole Normale Supérieure, \\ 24 Rue Lhomond, F-75231 Paris Cedex 05, France
}

October 26, 2018

\begin{abstract}
A pump-probe atomic parity violation (APV) experiment performed in a longitudinal electric field $\vec{E}_{l}$, has the advantage of providing a signal which breaks mirror symmetry but preserves cylindrical symmetry of the set-up, i.e. this signal remains invariant when the pump and probe linear polarizations are simultaneously rotated about their common direction of propagation. The excited vapor acts on the probe beam as a linear dichroic amplifier, imprinting a very specific signature on the detected signal. Our differential polarimeter is oriented to yield a null result unless a chirality of some kind is acting on the excited atoms. Ideally, only the APV ( $\vec{E}_{l}$-odd) and the calibration $\left(\vec{E}_{l}\right.$-even) signals should participate in such a chiral atomic response, a situation highly favourable to sensitive detection of a tiny effect. In the present work we give a thorough analysis of possible undesirable defects such as spurious transverse fields or misalignments, which may spoil the ideal configuration and generate a chiral response leading to possible systematics. We study a possible way to get rid of such defects by performing global rotations of the experiment by incremental angular steps $\phi$, leaving both stray fields and misalignments unaltered. Our analysis shows that at least two defects are necessary for the $\vec{E}_{l}$-odd polarimeter output to be affected; a $\cos (2 \phi)$ modulation in the global rotations reveals the transverse nature of the defects. The harmful systematic effects are those which subsist after we average over four configurations obtained by successive rotations of $45^{\circ}$. They require the presence of a stray transverse electric field. By doing auxiliary atomic measurements made in known, applied, magnetic fields which amplify the systematic effect, it is possible to measure the transverse E-field and to minimize it. Transverse magnetic fields must also be carefully compensated following a similar procedure. We discuss the feasibility of reducing the systematic uncertainty below the one percent level. We also propose statistical correlation tests as diagnoses of the aforementioned systematic effects.
\end{abstract}

PACS. 32.80.Ys - 32.60.+i - 33.55.Fi - 42.25.Lc

\footnotetext{
*Laboratoire de l'Ecole Normale Supérieure associé au CNRS (UMR 8552) et à l'Université Pierre et Marie Curie

${ }^{\dagger}$ Fédération de Recherche de l'Ecole Normale Supérieure associée au CNRS (FR684)
} 


\section{Introduction}

Atomic Parity violation (APV) experiments have been motivated by their ability to probe neutral current weak interactions in conditions very different from particle physics experiments of all kinds and hence to yield valuable complementary information [1. They probe the electron-quark electroweak interaction at distance scales very different from those explored in high energy measurements. Moreover, in atoms all the quarks contribute coherently while at high energies the nuclei and even the nucleons are broken and the quarks act independently. In atoms the issue at stake is the detection of a tiny electric dipole transition amplitude, strictly forbidden by the laws of electromagnetism, but allowed by the weak interaction which breaks mirror-symmetry. APV can "show up" in several ways: optical rotation in allowed $\mathrm{M}_{1}$ transitions [2, 3, 4, or electroweak interference effects in the transition probability of an $E$-field-assisted forbidden atomic transition [5], which affects either the population [6, 7, 8, 9, 10, or the orientation in the upper state [5]. In the latter case, the detection always relies on some fluorescence light monitoring with or without polarization analysis. Since the interference term involves the product of the parity violating amplitude $E_{1}^{p v}$ and the $E$-field induced amplitude, $\beta E$, while the transition rate is proportional to $|\beta E|^{2}$, the left-right asymmetry, $\propto \operatorname{Im} E_{1}^{p v} / \beta E$, decreases as $1 / E$. More recently, our group has demonstrated a novel kind of pump-probe experiment [11. Here the 6S-7S highly forbidden transition is excited in a longitudinal electric field $\vec{E}_{l}$ by an intense pulse of resonant light which lasts for a time shorter than the $7 \mathrm{~S}$ lifetime. It is immediately followed by the light pulse of a second beam, the probe, resonant with the $7 \mathrm{~S}-6 \mathrm{P}_{3 / 2}$ allowed transition and colinear with the excitation beam. For short pulse durations, the population inversion produced by the pump is sufficient to produce transient amplification of the probe beam. APV shows up because the probe amplification depends on the relative orientation of the linear polarization of the excitation laser $\hat{\epsilon}_{e x}$ and the probe laser $\hat{\epsilon}_{p r}$. Specifically, there is a chiral contribution to the optical gain of the vapor characterized by the pseudoscalar $\left(\hat{\epsilon}_{e x} \cdot \hat{\epsilon}_{p r}\right)\left(\hat{\epsilon}_{e x} \wedge \hat{\epsilon}_{p r} \cdot \vec{E}_{l}\right)$ which takes opposite values for two mirror-image configurations, as for instance those observed in the two channels of our polarimeter monitoring $\hat{\epsilon}_{p r}(\S 3)$. A 9\% accurate measurement [1], being currently improved, has validated the method. One of its advantages is to provide an independent method of APV measurement. It is well known that one of the main difficulties in measuring the very small APV effects lies in the discrimination against systematic effects. These have different origins depending on the chosen configuration, hence the importance of a new configuration. In addition, this stimulated emission detection scheme benefits from several attractive features: dark-field detection of the left-right asymmetry, reliable, line shape independent calibration procedure, amplification of the asymmetry itself. For instance, the right-left asymmetry instead of being a decreasing function of the applied $\vec{E}_{l}$-field benefits from an amplification mechanism via propagation of the probe beam through the optically thick excited vapor [12].

Moreover, the cylindrical symmetry of the experiment, another original feature, plays an important role: the amplification asymmetry is expected to remain invariant under simultaneous rotations of the polarizations $\hat{\epsilon}_{e x}$ and $\hat{\epsilon}_{p r}$ around their axis of propagation [13. In the present paper, we explain how this property can be exploited to discriminate against parity conserving (PC) signals generated by imperfections, because of their variation under these rotations. Although some of these signals simulate the PV effect in a given polarization configuration, their signatures are signals which break cylindrical symmetry. A schematic of the ideal experiment represented on Fig. 1 shows the two orthogonal symmetry planes defined by the electric field $\vec{E}_{l}$ and the linear excitation polarization $\hat{\epsilon}_{e x}$. APV gives rise to a tilt of the optical axes of the excited vapor out of those planes. The incoming probe polarization chosen either parallel or perpendicular to that of the excitation beam provides a 


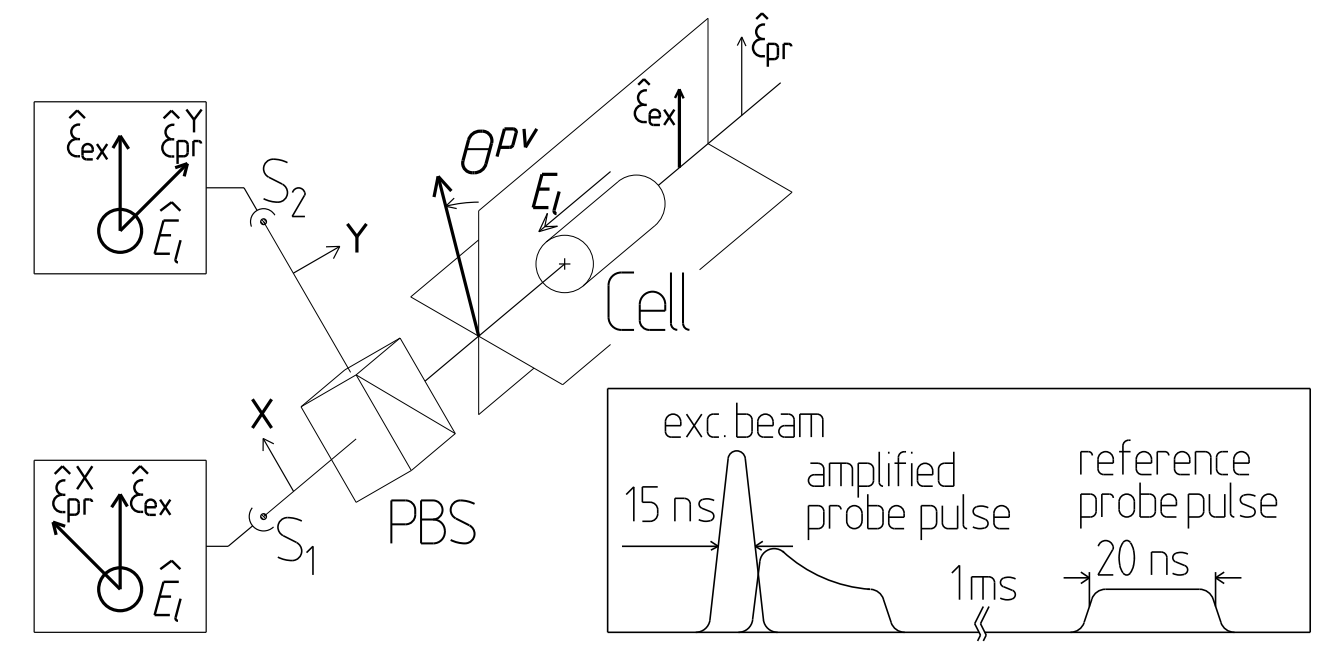

Figure 1: Schematic of the experiment showing the two orthogonal symmetry planes defined by the electric field $\vec{E}_{l}$ and the linear excitation polarization $\hat{\epsilon}_{e x}$. APV gives rise to a tilt $\theta^{p v}$ of the optical axes of the excited vapor out of these planes. The incoming probe polarization $\hat{\epsilon}_{p r}$ provides a superposition of the left and right-handed $\left(\hat{\epsilon}_{e x}, \hat{\epsilon}_{p r}^{X}, \vec{E}_{l}\right.$ and $\left.\hat{\epsilon}_{e x}, \hat{\epsilon}_{p r}^{Y}, \vec{E}_{l}\right)$ configurations analyzed. The probe amplification difference is directly extracted from the optical signals $\mathrm{S}_{1}, \mathrm{~S}_{2}$, recorded in each channel of the Polarizing Beam Splitter (PBS). Inset: timing of the experiment repeated at $100 \mathrm{~Hz}$.

superposition of the two configurations of opposite handedness, $\hat{\epsilon}_{e x}, \hat{\epsilon}_{p r}^{X}, \vec{E}_{l}$, and $\hat{\epsilon}_{e x}, \hat{\epsilon}_{p r}^{Y}, \vec{E}_{l}$, analyzed simultaneously by our polarimeter $\left(\hat{\epsilon}_{p r}^{X}\right.$ and $\hat{\epsilon}_{p r}^{Y}$ denoting the two components of the probe polarization at + and $-45^{\circ}$ to its input direction). Since our polarimeter operates in a balanced mode, the probe amplification difference, i.e. the right-left asymmetry, is directly extracted for each excitation laser pulse from the optical signals $S_{1}, S_{2}$, recorded for the two channels. The PV left-right asymmetry is expected to remain invariant under a global rotation of the experiment, performed by rotating the polarimeter and beam polarizations altogether around the common beam axis. This important test can reveal defects such as transverse $\vec{E}$ or $\vec{B}$ fields which remain fixed in the laboratory frame while the polarizations are rotated. We discuss how this can be used to minimize the systematic errors below a known level. This is even more important given that we are currently improving our experiment with the aim of reducing the statistical error down to the one percent level.

In this paper we first explain the origin of the chiral optical gain in terms of a parity violating contribution to the atomic alignment in the $7 \mathrm{~S}$ state induced by linearly polarized 6S-7S excitation $(\S 2)$. Next, we introduce a general formalism necessary for a rigorous treatment of experimental defects $(\S$. 3). The basic principle of our polarimetry measurements is presented and used to obtain the relation between the PV atomic alignment in the excited state and the "atomic" imbalance of the polarimeter (§. 3). This relation is useful to predict the variations of the signals generated by defects under simultaneous rotations $R(\hat{k}, \phi)$ of the input polarizations $\hat{\epsilon}_{p r}$ and $\hat{\epsilon}_{e x}$ about the common beam direction $\hat{k}$ by an angle $\phi$, which leave invariant the APV and the calibration signals. As an example we treat the perturbation induced by the simultaneous presence of transverse electric and magnetic fields, $\vec{E}_{t}$ and $\vec{B}_{t}$. We show that this leads to a parity conserving, magnetoelectric optical effect, which, depending on the property of $\vec{E}_{t}$ and $\vec{B}_{t}$ under the $\vec{E}_{l}$-field reversal, may simulate the PV signal in a fixed $\hat{\epsilon}_{e x}, \hat{\epsilon}_{p r}$ configuration, but which exhibits characteristic 
modulations under rotations of the polarizations (§. 4). In this particular case, it is easy to arrive at a judicious choice of the rotation angle $\phi$. We show that only four different $\hat{\epsilon}_{e x}, \hat{\epsilon}_{p r}$ configurations are necessary to extract maximal information about the systematic effect generated by the stray fields: namely those generated from an initial configuration by three steps of successive $45^{\circ}$ rotations of both $\hat{\epsilon}_{e x}$ and $\hat{\epsilon}_{p r}$. This result can be extended

to systematics arising from two transverse magnetic fields, one odd, and the other even in $\vec{E}_{l}$-reversal (§. 5). This property is further extended and can be associated to the most general structure of the atomic density matrix for the $7 \mathrm{~S}$ state. We show that a misalignment of the excitation and probe beams can have the same effect as a transverse magnetic field. Finally, taking into account the possible magnitude of the residual defects on our set-up, we discuss at what level we might reasonably expect to reduce the systematic uncertainty (§6.). In addition, we present a quite independent diagnosis based on a statistical test to be performed on the PV data which could reveal the presence of a harmful systematic effect of magnetoelectric origin.

\section{APV manifestation via a chiral optical gain}

\subsection{APV contribution to the angular anisotropy in the excited state}

When an atomic vapor is excited with a linearly polarized laser beam, resonant at the frequency of an $E_{1}$ allowed transition, it is easily verified that the excited state posseses an alignment with a privileged direction determined by the polarization of the excitation beam. More precisely the quantum average of the operator $\left|\vec{F} \cdot \hat{\epsilon}_{e x}\right|^{2}$ taken over the density matrix $\rho_{e}(t=0)$ of the $7 \mathrm{~S}, \mathrm{~F}^{\prime}$ state at the excitation time $t=0$, differs from the typical value for an isotropic distribution, i.e. $\operatorname{Tr}\left\{\rho_{e}(0)\left|\vec{F} \cdot \hat{\epsilon}_{e x}\right|^{2}\right\} \neq|\vec{F}|^{2} / 3 \cdot \operatorname{Tr}\left\{\rho_{e}(0)\right\}$.

Here we excite the vapor via one hyperfine component $6 S, F-7 S, F^{\prime}=F \pm 1$ of the highly forbidden transition. The effective transition dipole $\vec{d}^{\text {eff } f}$ can be written 11, 14:

$$
\vec{d}^{e f f}=-i \beta \vec{\sigma} \wedge \vec{E}_{l}+M_{1} \vec{\sigma} \wedge \hat{k}-i \operatorname{Im} E_{1}^{p v} \vec{\sigma} .
$$

The components of the electronic spin operator $\vec{\sigma}$ are the Pauli spin matrices. The first contribution is the parity conserving (PC) amplitude induced by the applied electric field parallel to the wave vector $\hat{k} \| \hat{z}$ of the excitation beam and associated with the vector part of the transition polarizability $\beta$. The scalar part $\vec{d}=-\alpha \vec{E}$ can be ignored on a $F \rightarrow F^{\prime}=F \pm 1$ transition. Hyperfine mixing in presence of a transverse magnetic field will be considered in $\S 4.5$. The second contribution is associated with the $M_{1}$ amplitude, but it will be shown later on $(\S 2.4)$ to lead only to very small effects. The last term in Eq. 1 arises from the PV electric dipole amplitude $E_{1}^{p v}$ characteristic of the weak interaction. If we ignore for the moment the $M_{1}$ contribution and if we choose $\vec{E}_{l}= \pm E_{l} \hat{z}$, we can rewrite the transition amplitude as follows:

$$
\vec{d}^{e f f} \cdot \hat{\epsilon}_{e x}=\mp i \beta E_{l} \vec{\sigma} \wedge \hat{z} \cdot\left(\hat{\epsilon}_{e x} \pm \theta^{p v} \hat{z} \wedge \hat{\epsilon}_{e x}\right), \quad \text { with } \quad \theta^{p v}=-\operatorname{Im} E_{1}^{p v} / \beta E_{l} .
$$

This equation has a simple physical interpretation: the effect of the PV transition amplitude is equivalent to a rotation of the linear polarization $\hat{\epsilon}_{e x}$ about $\vec{E}_{l}$ by the small angle $\theta^{p v}$, typically $\sim 10^{-6} \mathrm{rad}$ for $E_{l} \approx 1.7 \mathrm{kV} / \mathrm{cm}$. The sense of this rotation changes when the direction of $\vec{E}_{l}$ is reversed. The angle $\theta^{p v}$ is the important parameter to be determined since it yields the ratio between the PV and the Stark amplitudes. Provided the $E_{l}$-field magnitude is known, one can use a value of $\theta^{p v}$ to obtain the weak charge $Q_{W}$ of the Cs nucleus by relying on Atomic Physics calculations of $E_{1}^{p v} / Q_{W}$ [15, 16, 17, 18]. 


\section{$2.2 \quad$ Alignment tensor of the excited state.}

The Stark-induced transition creates an excited state $7 S, F^{\prime}$ endowed with an alignment. For the sake of completeness we summarize here some basic definitions. First we introduce the second-rank tensor operator $\hat{T}^{(2)}$ constructed from the total angular momentum $\vec{F}$. In order to avoid unnecessary algebraic complications we shall use a cartesian basis set:

$$
\hat{T}_{i j}^{(2)}=\frac{1}{2}\left(F_{i} F_{j}+F_{j} F_{i}\right)-\frac{1}{3} \vec{F}^{2} \delta_{i, j} .
$$

We define now the traceless symmetric alignment tensor $\mathcal{A}_{i j}$ by the following quantum averages :

$$
\mathcal{A}_{i j}(t)=\operatorname{Tr}\left\{\rho(t) \hat{T}_{i j}^{(2)}\right\}, \quad \text { implying } \quad \sum_{k} \mathcal{A}_{k k}(t)=0 .
$$

It is convenient to introduce as a visual tool the alignment ellipsoid defined by the quadratic equation :

$$
\sum_{i, j} x_{i} x_{j}\left(\mathcal{A}_{i j}+\frac{1}{3} F^{\prime}\left(F^{\prime}+1\right) \delta_{i, j}\right)=\frac{1}{3} F^{\prime}\left(F^{\prime}+1\right) .
$$

In absence of alignment $\left(\mathcal{A}_{i j}=0\right)$ the ellipsoid reduces to the unit sphere.

\subsubsection{General expression}

For the Stark-induced transition induced by the vector polarizability, in contrast to allowed electric dipole ones, the preferred direction of the excitation process is not $\hat{\epsilon}_{e x}$ but rather the orthogonal direction $\hat{E}_{l} \wedge \hat{\epsilon}_{e x}$. If we ignore the PV contribution, the alignment created in the excited state can be represented by an ellipsoid of revolution symmetry about a direction parallel to $\hat{k} \wedge \hat{\epsilon}_{e x}$ which preserves the planes of symmetry of the experiment defined by the common direction of $\vec{E}_{l}$ and $\hat{k}$ and by $\hat{\epsilon}_{e x}$ (see Fig. 1). The effect of the PV contribution is to tilt this ellipsoid about $\vec{E}_{l}$ by the small angle $\theta^{p v}$. As a result, the alignment no longer preserves the symmetry planes of the experiment: this is the manifestation of parity violation in the pump-probe experiment discussed presently.

We are going to present a formalism which can be applied to more general situations than the Stark-induced $6 S \rightarrow 7 S$ transition and which will allow us to incorporate the $E_{1}^{p v}$ and $M_{1}$ contributions as well as those associated with experimental defects breaking the cylindrical symmetry of the ideal experiment. The effective transition operator $T_{\text {eff }}$ is then given by:

$$
\begin{aligned}
T_{\text {eff }} & =\vec{b} \cdot \vec{\sigma} \\
\text { where } \vec{b} & =i \beta \vec{E} \wedge \hat{\epsilon}_{e x}+i \operatorname{Im} E_{1}^{p v} \hat{\epsilon}_{e x}-M_{1} \hat{k} \wedge \hat{\epsilon}_{e x} .
\end{aligned}
$$

The direction of $\vec{E}$ is for the moment arbitrary. The laser selects only one hfs component $n S, F \rightarrow(n+1) S, F^{\prime}$. The excited state density matrix, up to a normalization factor, is then given by:

$$
\rho_{e}=P_{F^{\prime}} T_{e f f} P_{F} \rho_{g} P_{F} T_{e f f}^{\dagger} P_{F^{\prime}},
$$

where $\rho_{g}$ is the restriction of the density operator to the $n S$ ground state. $P_{F}$ is the projector on the $n S, F$ sublevel and $P_{F^{\prime}}$ the projector on the $(n+1) S, F^{\prime}$ sublevel. Since we are mostly interested in the $6 S, F \rightarrow 7 S, F^{\prime}$ transition with $F^{\prime} \neq F$, it is convenient to write $\rho_{g}=P_{F}$ and $P_{F^{\prime}}=\mathbb{I}-P_{F}$. We apply the Wigner-Eckart theorem to the spin operator $\vec{\sigma}$ acting in the hyperfine subspace $F$ :

$$
P_{F} \vec{\sigma} P_{F}=2 g_{F} P_{F} \vec{F}, \quad \text { where } \quad g_{F}=-g_{F^{\prime}}=2(F-I) /(2 I+1),
$$


and $I$ is the nuclear spin, in our case equal to $7 / 2$ for natural cesium, ${ }^{133} \mathrm{Cs}$.

Using the following identities involving the $c$-number vector $\vec{b}$ :

$$
P_{F^{\prime}} \vec{\sigma} \cdot \vec{b} P_{F} \vec{\sigma} \cdot \vec{b}^{*} P_{F}^{\prime}=P_{F^{\prime}} \vec{\sigma} \cdot \vec{b}\left(1-P_{F^{\prime}}\right) \vec{\sigma} \cdot \vec{b}^{*} P_{F^{\prime}}=P_{F^{\prime}}|\vec{b}|^{2}-P_{F^{\prime}} \vec{\sigma} \cdot \vec{b} P_{F^{\prime}} \vec{\sigma} \cdot \vec{b}^{*} P_{F^{\prime}},
$$

we readily obtain the excited state density matrix $\rho_{e}(0)$ at the excitation time $t=0$ :

$$
\rho_{e}(0)=\left(|\vec{b}|^{2}-4 g_{F^{\prime}}^{2}(\vec{F} \cdot \vec{b})\left(\vec{F} \cdot \vec{b}^{*}\right)\right) P_{F^{\prime}} .
$$

Later on, it will be of interest to consider the transformation of the excited state density matrix under a space rotation $R: \rho_{e}(0) \rightarrow U(R) \rho_{e}(0) U(R)^{\dagger}$, where $U(R)$ is the unitary operator associated to $R$. Using the following basic relations which result from the very definition of $U(R)$, we can write $U(R) \vec{b} \cdot \vec{F} U(R)^{\dagger}=\left(R^{-1} \vec{F}\right) \cdot \vec{b}=\vec{F} \cdot(R \vec{b})$, we arrive to a simple rule for the density matrix rotation transformation : on the r.h.s of eq.(8) replace the vectors $\vec{b}$ and $\vec{b}^{*}$ by the rotated vectors: $\vec{b} \rightarrow R \vec{b}, \vec{b}^{*} \rightarrow R \vec{b}^{*}$.

Let us now evaluate the $7 S$ alignment tensor components $\mathcal{A}_{i j}^{e}(0)$ :

$$
\begin{aligned}
\mathcal{A}_{i j}^{e}(0) & =\operatorname{Tr}\left\{\rho_{e}(0)\left(F_{i} F_{j}-\frac{F^{\prime}\left(F^{\prime}+1\right)}{3} \delta_{i, j}\right)\right\} \\
& =-4 g_{F^{\prime}}^{2}\left(\operatorname{Tr}\left\{(\vec{F} \cdot \vec{b})\left(\vec{F} \cdot \vec{b}^{*}\right) F_{i} F_{j} P_{F^{\prime}}\right\}-|\vec{b}|^{2}\left(2 F^{\prime}+1\right)\left(\frac{F^{\prime}\left(F^{\prime}+1\right)}{3}\right)^{2} \delta_{i, j}\right)
\end{aligned}
$$

In order to proceed, we have to extract from the rank-four tensor operator $T_{i j k l}=$ $F_{i} F_{j} F_{k} F_{l}$ the scalar pieces which are the only ones having non-zero traces. They are obtained by contracting two pairs among the four indices in all possible ways. We arrive in this manner at the following expression for the needed trace, where we have used its invariance under circular permutation of the indices :

$$
\operatorname{Tr}\left\{T_{i j k l}\right\}=A\left(\delta_{i, j} \delta_{k, l}+\delta_{i, l} \delta_{j, k}\right)+B \delta_{i, k} \delta_{j, l} .
$$

The rational numbers $A$ and $B$ can be easily obtained as linear combinations of $S_{2}\left(F^{\prime}\right)$ and $S_{4}\left(F^{\prime}\right)$ where $S_{n}(N)=\sum_{m=0}^{m=N} m^{n}$ by calculating directly $\operatorname{Tr} F_{z}^{2}$ and $\operatorname{Tr} F_{z}^{4}$ and comparing with the results obtained by using eq. (10):

$$
\begin{aligned}
& A=F^{\prime}\left(F^{\prime}+1\right) S_{2}\left(F^{\prime}\right)-S_{4}\left(F^{\prime}\right)=\frac{1}{30} F^{\prime}\left(F^{\prime}+1\right)\left(2 F^{\prime}+1\right)\left(2 F^{\prime 2}+2 F^{\prime}+1\right) \\
& B=4 S_{4}\left(F^{\prime}\right)-2 F^{\prime}\left(F^{\prime}+1\right) S_{2}\left(F^{\prime}\right)=\frac{1}{15}\left(F^{\prime}-1\right) F^{\prime}\left(F^{\prime}+1\right)\left(2 F^{\prime}+1\right)\left(F^{\prime}+2\right) .(11)
\end{aligned}
$$

We have now all we need to compute the alignment tensor $\mathcal{A}_{i j}^{e}(0)$, which is expected to be proportional to the traceless second-rank, symmetric, real tensor built from the complex vector $\vec{b}$ :

$$
\mathcal{A}_{i j}^{e}(0)=-4 g_{F^{\prime}}^{2} \mathcal{F}\left(\frac{1}{2}\left(b_{i} b_{j}^{*}+b_{i}^{*} b_{j}\right)-\frac{1}{3}|\vec{b}|^{2} \delta_{i, j}\right),
$$

where the angular momentum factor $\mathcal{F}$ is given by:

$$
\mathcal{F}=A+B=\frac{1}{30} F^{\prime}\left(F^{\prime}+1\right)\left(2 F^{\prime}-1\right)\left(2 F^{\prime}+1\right)\left(2 F^{\prime}+3\right) .
$$




\subsubsection{The Stark alignment tensor in the ideal longitudinal configuration}

We are going to apply the formula (12) to the Stark amplitude which is always the dominant one in realistic experimental situations. In the ideal experiment the applied electric field $\vec{E}_{l}$ is taken colinear with the wave vector $\vec{k}$, i.e. $\vec{E}_{l} \wedge \vec{k}=0$. The vector $\vec{b}$ is then given by:

$$
\vec{b}_{S t}=i \beta E_{l} \hat{E}_{l} \wedge \hat{\epsilon}_{e x}=i \beta E_{l} \hat{h}, \quad \text { with } \quad \hat{h}=\hat{E}_{l} \wedge \hat{\epsilon}_{e x} .
$$

In this paper to a given vector $\vec{v}$ there corresponds a unit vector $\hat{v}=\vec{v} / v$ with $v=|\vec{v}|$.

We get then immediately the Stark-induced alignment tensor by inserting $\vec{b}_{S t}$ in Eq. (12):

$$
\mathcal{A}_{i j}^{S t}(0)=-4 g_{F^{\prime}}^{2} \beta^{2} E_{l}^{2} \mathcal{F}\left(\hat{h}_{i} \hat{h}_{j}-\frac{1}{3} \delta_{i, j}\right) .
$$

\subsubsection{PV effect on the Stark alignment tensor}

To get the first-order PV correction $\Delta_{p v} \mathcal{A}_{i j}(0)$ to the Stark alignment tensor we perform a first order expansion of $\mathcal{A}_{i j}^{e}(0)$ by writing $\vec{b}=\vec{b}_{S t}+\Delta_{p v} \vec{b}$ with $\Delta_{p v} \vec{b}=i \operatorname{Im} E_{1}^{p v} \hat{\epsilon}_{e x}$. Let us now insert in formula (12) the first order correction to $b_{i} b_{j}$ :

$$
\left.\left.\Delta_{p v} b_{i} b_{j}=\left(b_{S t}\right)_{i} \Delta_{p v} b_{j}^{*}+b_{S t}\right)_{i}^{*} \Delta_{p v} b_{j}\right)=\beta E_{l} \operatorname{Im} E_{1}^{p v}\left(\hat{h}_{i}\left(\hat{\epsilon}_{e x}\right)_{j}+(i \leftrightarrow j)\right) .
$$

We get immediately the PV correction to the alignment tensor:

$$
\Delta_{p v} \mathcal{A}_{i j}^{e}(0)=-4 g_{F^{\prime}}^{2} \beta^{2} E_{l}^{2} \mathcal{F} \theta_{p v}\left(\left(\hat{E}_{l} \wedge \hat{\epsilon}_{e x}\right)_{i}\left(\hat{\epsilon}_{e x}\right)_{j}+(i \leftrightarrow j)\right) .
$$

As we have already noted, the effect of the Stark- $E_{1}^{p v}$ amplitude is to rotate the alignment ellipsoid by a small angle $\theta^{p v}$ about the direction of $\hat{E}_{l}$. The alignment tensor is then expected to be subjected to the same infinitesimal rotation. This result is easily verified by showing that $\Delta_{p v} \vec{b} \equiv i \operatorname{Im} E_{1}^{p v} \hat{\epsilon}_{e x}=\theta^{p v} \hat{E}_{l} \wedge \vec{b}_{S t}$, this following immediately from the identities:

$$
\theta^{p v}=-\operatorname{Im} E_{1}^{p v} / \beta E_{l} \quad \text { and } \quad \hat{E}_{l} \wedge\left(\hat{E}_{l} \wedge \hat{\epsilon}_{e x}\right)=-\hat{\epsilon}_{e x} .
$$

\subsubsection{Absence of alignment induced by the Stark- $M_{1}$ interference.}

The $M_{1}$ contribution to the vector $\vec{b}$ appearing in Eq.(5) is given by $\vec{b}_{M_{1}}=M_{1} \hat{k} \wedge \hat{\epsilon}_{e x}$. The crucial point is that $\vec{b}_{M_{1}}$ is a purely real vector, while $\vec{b}_{S t}$ is an imaginary one. It follows immediately that the Stark- $M_{1}$ interference contribution to $\frac{1}{2}\left(b_{i} b_{j}^{*}+b_{i}^{*} b_{j}\right)=\operatorname{Re}\left(b_{i} b_{j}^{*}\right)$ vanishes since $\left(b_{S t}\right)_{i}\left(b_{M_{1}}\right)_{j}^{*}+\left(b_{M_{1}}\right)_{i}\left(b_{S t}\right)_{j}^{*}$ is clearly imaginary. In conclusion, the Stark- $M_{1}$ interference term does not contribute to the alignment tensor within the hypotheses leading to $E q$. (12) . A crucial one of these concerns the $6 S, F$ density matrix which is assumed to be a thermal distribution: $\rho_{g} \propto P_{F}$. This property does not hold in presence of a static magnetic field $\vec{B}$, if the excitation beam intensity varies significantly within a frequency interval of the order of the Zeeman splittings. We will show later on, that if $\vec{E}$ has a component transverse to $\vec{k}$, then the Stark- $M_{1}$ interference leads to a non-zero alignment contribution.

It is worth stressing the importance of the result obtained in this section. The $M_{1^{-}}$ Stark interference is a potential source of systematics which has required special care in all experiments performed on highly forbidden transitions in the transverse field configuration so far [5] 10. Thus the absence of $M_{1}$-Stark interference in the longitudinal field configuration examined here is a significant advantage. In this configuration, it is clear that the $\pi / 2$ phase difference between the $M_{1}$ and the Stark amplitudes prevents there being any interference 
whatever the excitation polarization, and this even if the atomic system is perturbed by a magnetic field.

It is only in presence of a stray transverse electric field that we shall have to consider the possibility of systematic effects associated with the magnetic dipole amplitude.

\subsection{Optical anisotropy resulting from the APV alignment}

While the population inversion causes an amplification of the probe beam, its polarization is altered by the angular anisotropy created in the excited state. We obtain the atomic anisotropies in the excited state by detecting the optical anisotropies that they induce on the amplified probe beam. Here the vapor is endowed with an atomic alignment. Associated with the alignment ellipsoid is an ellipsoid of refractive index for light resonant with one hyperfine component of the $7 S-6 P_{3 / 2}$ transition. The relative magnitude of the axes

depends on the hyperfine component. The eigenaxes are those of the ellipse resulting from the intersection of the alignment ellipsoid by a plane orthogonal to the wave vector $\hat{k}$ of the probe beam. The imaginary part of the refractive index is responsible for the gain which takes two different values depending on whether the linear polarization $\hat{\epsilon}_{p r}$ is aligned along one or the other eigenaxis, an effect generally dubbed linear dichroism, while the real part is responsible for birefringence of the vapor. For the probe beam which propagates along $\hat{z}$, the Stark and the PV alignments both induce a linear dichroism, but the former with axes along $\hat{\epsilon}_{e x}$ and $\hat{E}_{l} \wedge \hat{\epsilon}_{e x}$ and the latter with axes $\hat{X}$ and $\hat{Y}$. It is this latter contribution, the optical anisotropy resulting from the APV alignment, which gives rise to the APV signal in the pump-probe experiment. As a consequence the global gain matrix of the excited vapor has its axes tilted with respect to $\hat{\epsilon}_{e x}$ by the tiny angle $\theta^{p v}$. The tilt is opposite for two mirror-image configurations associated with opposite signs of the pseudoscalar $\mathcal{S}^{c h}=\left(\hat{\epsilon}_{e x} \cdot \hat{\epsilon}_{p r}\right)\left(\hat{\epsilon}_{e x} \wedge \hat{\epsilon}_{p r} \cdot \vec{E}_{l}\right)$. Hence the notion of a chiral optical gain. The probe polarization aligned along $\hat{\epsilon}_{e x}$ at the cell entrance actually does not lie along an eigenaxis. Consequently its direction is modified during the propagation of the probe beam through the vapor. This modification is measured using polarimetry methods which allow us to obtain an absolute determination of $\theta^{p v}$, as explained in the next section.

\subsection{Absolute calibration of the tilt angle $\theta^{p v}$}

The physical interpretation of APV given in $\S 2.1$ suggests a natural method of calibration. The APV contribution can be seen as resulting from the dominant PC contribution via an infinitesimal rotation of angle $\theta^{p v}$ about $\hat{E}_{l}$. By performing small rotations of the excitation polarization by a known angle $\theta_{c a l}$, while keeping $\hat{\epsilon}_{p r}$ unchanged at the input, we can induce an optical anisotropy for the probe exactly similar to the APV one, eventhough it is P-conserving. The ratio of these two effects, measured under identical conditions and distinguished by their opposite dependence on $\theta_{c a l}$ and $E_{l}$ reversals, yields directly $\theta^{p v} / \theta_{c a l}$. By measuring the ratio, it is possible to eliminate different effects affecting the magnitude of each signal individually which would otherwise be difficult to predict quantitatively (see $\S 3.2)$. 


\section{Balanced-mode polarimetry measurements on the amplified probe beam}

\subsection{Operating conditions providing polarimeter imbalances insen- sitive to the PC alignment}

Since we want to measure a small PV anisotropy in presence of a large PC one, we orient the polarimeter so that it is insensitive to the latter, and fully sensitive to the former. We have chosen a polarization beam splitter cube having its axes $\hat{X}$ and $\hat{Y}$ oriented at $\pm 45^{\circ}$ to the excitation and probe polarizations. The input probe polarization is a superposition of the two eigenpolarizations, $\hat{\epsilon}_{X}$ and $\hat{\epsilon}_{Y}$ of the polarimeter:

$$
\hat{\epsilon}_{p r}=\left(\hat{\epsilon}_{X}+\hat{\epsilon}_{Y}\right) / \sqrt{2} \text {. }
$$

In absence of the excitation beam, the electronic gains of the two channels are adjusted to ensure equality of the probe signals detected in both channels, $S_{X}=S_{Y}$. However, due to possible drifts in the electronic or optical components, one has to isolate the true atomic contribution to the imbalance. For each excitation laser pulse, the imbalance for the amplified probe pulse, $D^{a m p}=\frac{\left(S_{X}-S_{Y}\right)}{\left(S_{X}+S_{Y}\right)}$ is compared with a reference value $D^{r e f}$, measured when all the $7 S$ excited atoms have decayed. Thus the doubly differential signal, $D^{a m p}-D^{r e f}$, selects at the laser repetition rate a true atomic effect, $D^{a t}$, free of the polarization defects present on the probe beam path.

\subsection{Relation between atomic alignment and polarimeter imbalance}

Since the probe polarization is adjusted at cell entrance to be parallel to $\hat{\epsilon}_{e x}=\hat{y}$ which (without APV) defines the eigenaxes of the vapor gain, it should remain parallel to one eigen axis of the gain during propagation through the vapor. An atomic imbalance is expected to arise specifically from a difference of light amplification for a probe beam polarized along $\hat{X}$ or $\hat{Y}$.

In a previous publication (see [20] Eq. 20 or $\S 5.2$ ) we have calculated the intensity and polarization modification of a linearly polarized probe beam resonant for a single hyperfine component of the probe transition $7 S_{1 / 2} \rightarrow 6 P_{3 / 2}$. Following a similar approach, we shall write the light intensity difference $S_{X}-S_{Y}$ as an expression proportional to the contraction of the alignment tensor $\mathcal{A}_{i j}^{e}(\tau)$ - at the time of detection - with the traceless symmetric tensor $\mathcal{D}_{i j}$ which characterizes the differential two-channel polarimeter:

$$
\mathcal{D}_{i j}=\left(\hat{\epsilon}_{X}\right)_{i}\left(\hat{\epsilon}_{X}\right)_{j}-\left(\hat{\epsilon}_{Y}\right)_{i}\left(\hat{\epsilon}_{Y}\right)_{j}
$$

Let us denote by $\vec{D}$ the electric dipole moment operator and by $\vec{d}$ se the effective dipole operator which induces specifically the stimulated emission transition $7 S, F^{\prime} \rightarrow 6 P_{3 / 2}, F_{p}$ :

$$
\vec{d}^{s e}=P_{F_{p}} \vec{D} P_{F^{\prime}}
$$

where $P_{F_{p}}$ is the projection operator on the $6 P_{3 / 2}, F_{p}$ sublevel while $P_{F^{\prime}}$ is relative to the $7 S, F^{\prime}$ sublevel. (Note that $\overrightarrow{d^{s e}}$ is non-hermitian, but still a vector operator.) Now let us introduce the second-rank tensor operator: $\mathcal{O}_{i j}=\left(d_{i}^{s e}\right)^{\dagger} d_{j}^{s e}$ whose action is restricted to the $7 S, F^{\prime}$ subspace. Applying the Wigner-Eckart theorem, we can write:

$$
\mathcal{O}_{i j}=\left(d_{i}^{s e}\right)^{\dagger} d_{j}^{s e}=C_{s e}\left(6 P_{3 / 2} F_{p} \mid 7 S F^{\prime}\right) F_{i} F_{j} P_{F^{\prime}},
$$


where $C_{s e}\left(6 P_{3 / 2} F_{p} \mid 7 S F^{\prime}\right)$ is a real constant (explicitely calculated in [20]).

We now have all the tools to obtain the result announced above.

$$
\begin{aligned}
S_{X}-S_{Y} & \propto \operatorname{Tr}\left\{\rho_{e}(\tau)\left(\vec{d}^{s e} \cdot \hat{\epsilon}_{X}\right)^{\dagger}\left(\vec{d}^{s e} \cdot \hat{\epsilon}_{X}\right)-(X \rightarrow Y)\right\} \\
& \propto \mathcal{D}_{i j} \cdot \operatorname{Tr}\left\{\rho_{e}(\tau) \mathcal{O}_{i j}\right\} \propto \mathcal{D}_{i j} \cdot \mathcal{A}_{i j}^{e}(\tau),
\end{aligned}
$$

where the dot indicates that the two tensors have to be contracted, i.e. summations have to be performed upon the repeated indices.

Let us calculate the imbalance signal $S_{X}-S_{Y}$ using the alignment tensors $\mathcal{A}_{i j}^{e}$ derived in the previous section and given in equations (14) and (15). We deal first with the Starkinduced alignment:

$S_{X}-S_{Y} \propto \mathcal{D}_{i j} \cdot \mathcal{A}_{i j}^{S t}(0)=-4 g_{F^{\prime}}^{2} \beta^{2} E_{l}^{2} \mathcal{F} \mathcal{D}_{i j} \cdot \hat{h}_{i} \hat{h}_{j}=-4 g_{F^{\prime}}^{2} \beta^{2} E_{l}^{2} \mathcal{F}\left(\left(\hat{\epsilon}_{X} \cdot \hat{h}\right)^{2}-\left(\hat{\epsilon}_{Y} \cdot \hat{h}\right)^{2}\right)=0$.

The above cancellation follows immediately from the two-channel polarimeter eigenaxis expressions in a defect-free experimental set up :

$$
\hat{\epsilon}_{X, Y}=\frac{1}{\sqrt{2}}\left(\hat{\epsilon}_{e x} \pm \hat{\epsilon}_{e x}^{\perp}\right) \quad \text { with } \quad \hat{\epsilon}_{e x}^{\perp}=\hat{z} \wedge \hat{\epsilon}_{e x}
$$

$\hat{z}$ being a direction colinear to $\hat{k}$ and $\hat{E}_{l}$ which remains fixed in the laboratory whatever parameter reversal is made. This obviously implies: $\hat{\epsilon}_{X} \cdot \hat{h}=-\hat{\epsilon}_{Y} \cdot \hat{h}$.

In absence of defects, such as deliberate or accidental optical misalignements, stray electric and magnetic fields - to be discussed extensively in the next section - the only possible source for non-zero polarimeter imbalance will turn out to be the PV alignement calculated in the previous section (see Eq.(15)). Let us insert $\Delta_{p v} \mathcal{A}_{i j}^{e}(0)$ in the formula (19) giving $S_{X}-S_{Y}$ in terms of the alignment tensor:

$$
\begin{aligned}
\left(S_{X}-S_{Y}\right)_{p v} & \propto \mathcal{D}_{i j} \cdot \Delta_{p v} \mathcal{A}_{i j}^{e}(0)=-4 g_{F^{\prime}}^{2} \beta^{2} E_{l}^{2} \theta_{p v} \mathcal{F} \mathcal{D}_{i j} \cdot\left(\hat{h}_{i}\left(\hat{\epsilon}_{e x}\right)_{j}+(i \leftrightarrow j)\right) \\
& =-4 g_{F^{\prime}}^{2} \beta^{2} E_{l}^{2} \theta_{p v} \mathcal{F}\left(\left(\hat{h} \cdot \hat{\epsilon}_{X}\right)\left(\hat{\epsilon}_{e x} \cdot \hat{\epsilon}_{X}\right)-(X \rightarrow Y)\right) \\
& =-4 g_{F^{\prime}}^{2} \beta^{2} E_{l}^{2} \theta_{p v} \mathcal{F}\left(\left(\hat{E}_{l} \wedge \hat{\epsilon}_{e x} \cdot \hat{\epsilon}_{X}\right)\left(\hat{\epsilon}_{e x} \cdot \hat{\epsilon}_{X}\right)-(X \rightarrow Y)\right) .
\end{aligned}
$$

In the above formula appears, as expected, pseudo-scalars built from the physical vector objects involved in the experiment namely the longitudinal field $\vec{E}_{l}$, the laser polarization $\hat{\epsilon}_{e x}$ and the polarimeter eigenaxes:

$$
\mathcal{S}_{\text {chir }}\left(\hat{\epsilon}_{X}, \hat{E}_{l}\right)=\left(\hat{E}_{l} \wedge \hat{\epsilon}_{e x} \cdot \hat{\epsilon}_{X}\right)\left(\hat{\epsilon}_{e x} \cdot \hat{\epsilon}_{X}\right)=-\mathcal{S}_{\text {chir }}\left(\hat{\epsilon}_{Y}, \hat{E}_{l}\right) .
$$

Note that the last equality reflects the fact that we obtain $\hat{\epsilon}_{Y}$ from $\hat{\epsilon}_{X}$ by performing a mirror reflexion with respect to the plane containing $\hat{\epsilon}_{e x}$ and $\vec{E}_{l}$.

All of this means that the imbalance $D^{a t}=\left(S_{X}-S_{Y}\right) /\left(S_{X}+S_{Y}\right)$ allows one to search for the tiny PV effect using dark field detection. In a general way such an atom-induced imbalance should vanish unless some chirality is present in the experiment.

In Eq.(19), $\tau$ is the instant of detection which differs from the instant of excitation taken for origin of time. This means that in presence of a magnetic field we have to take into account the evolution of the excited state over the time interval which separates the instants of excitation and stimulated emission.

We can write the general relation:

$$
D^{a t}=K \mathcal{D}_{i j} \cdot \mathcal{A}_{i j}(\tau) .
$$


Several effects are embodied in the evaluation of the proportionality coefficient $K$ : not only transition probabilities depending on the hyperfine quantum numbers of the hyperfine states connected by the probe transition 20, but also the probe pulse time and duration, the optical thickness of the vapor at the probe laser wavelength as well as saturation effects induced by the probe beam [21]. The calculation of $D^{a t}$ involves the problem of the amplification of the probe while it propagates through the vapor. This has been considered previously both theoretically [21] and experimentally [12]. We refer the reader to previous work for the evaluation of the amplification factor common to both $S_{X}$ and $S_{Y}$ as well as the asymmetry amplification which affects directly the difference $S_{X}-S_{Y}$ and thereby the atomic imbalance $D^{a t}$. The crucial point here is that our measurement method includes a calibration procedure allowing us to eliminate $K$ by performing ratios between atomic imbalances of different physical origin but similar optical properties. To do this we apply a small rotation of $\hat{\epsilon}_{e x}$ of angle $\theta_{\text {cal }}$ around $\hat{z}$ before entrance in the cell, the sense of this rotation being independent of the direction of $\hat{E}_{l}$. The calculation of the corresponding correction to the alignment tensor mathematically is identical to that of $\Delta_{p v} \mathcal{A}_{i j}(0)$ except for the trivial replacement $\theta^{p v} \hat{E}_{l} \rightarrow \theta_{\text {cal }} \hat{z}$. This yields the atomic imbalance:

$$
D^{a t}\left(\theta_{\text {cal }}-\text { odd }, E_{l}-\text { even }\right)=-8 g_{F^{\prime}}^{2} \beta^{2} E_{l}^{2} \theta_{\text {cal }} K \mathcal{F} \mathcal{S}_{\text {chir }}\left(\hat{\epsilon}_{X}, \hat{E}_{l}\right)\left(\hat{z} \cdot \hat{E}_{l}\right),
$$

with which we compare the atomic APV imbalance:

$$
D^{a t}\left(\theta_{\text {cal }}-\text { even, } E_{l}-\text { odd }\right)=-8 g_{F^{\prime}}^{2} \beta^{2} E_{l}^{2} \theta^{p v} K \mathcal{F} \mathcal{S}_{\text {chir }}\left(\hat{\epsilon}_{X}, \hat{E}_{l}\right) \text {, }
$$

allowing us to calibrate $\theta^{p v}$ in terms of the known angle $\theta_{c a l}$. All the atomic factors embodied in $K$, as well as $E_{l}^{2}$ are eliminated in the ratio of both imbalances, provided they are measured under identical conditions. As long as this important condition is fulfilled, the relation

$$
\frac{D^{a t}\left(\theta_{c a l}-\text { even }, E_{l}-o d d\right)}{D^{a t}\left(\theta_{c a l}-o d d, E_{l}-e v e n\right)}=\frac{\theta^{p v}}{\theta_{c a l}}\left(\hat{z} \cdot \hat{E}_{l}\right)
$$

holds whatever the detection conditions, for any hyperfine component. The factor $\left(\hat{z} \cdot \hat{E}_{l}\right)=$ \pm 1 , depending on the sign of the projection of $\vec{E}_{l}$ on the fixed direction $\hat{z}$, will appear frequently in the forthcoming formulae.

\subsection{Symmetry breaking associated with a $B_{z}\left(E_{l}-o d d\right)$ magnetic field}

Since it is a pseudo-vector, a longitudinal magnetic field,breaks the mirror reflexion symmetry with respect to the plane containing $\hat{\epsilon}_{e x}$ and $\vec{E}_{l}$. It does not however break the cylindrical symmetry of the experiment. Therefore, it escapes the methods of diagnosis based on global rotations of the experiment, discussed in the present paper. This effect discussed previously 23] is quoted here just for completeness.

A magnetic field $B_{z}$ causes Larmor precession of the angular momentum $\vec{F}$ about the $\hat{z}$ axis for the average duration $\tau$ which separates the time of stimulated emission from the time of excitation. Consequently, after evolution of the density matrix in the excited state, the axes of the Stark alignment $\mathcal{A}_{i j}(\tau)$ no longer lie in the symmetry planes. They deviate by an angle $\gamma B_{z} \tau$ (with $\gamma B_{z}=\omega_{F}$, being the angular precession frequency). This effect is exactly similar to the calibration effect provided the angle $\theta_{\text {cal }}$ be replaced by $\gamma B_{z} \tau$. A problem arises only if the $B_{z}$ field is odd under $\vec{E}_{l}$ reversal, in which case there arises a signal simulating exactly the PV effect. To account for it, we measure the magnitude of the $B_{z}$-odd field seen by the atoms. This is performed by measuring the optical rotation (see $\S 4.3 .4$ ) induced by $B_{z}$-odd on a probe transition which is particularly sensitive $7 S, F=4 \rightarrow 6 P_{3 / 2}, F=5$ [24]. In our past measurements [11] it has vanished part of the time and never exceeded $50 \mu \mathrm{G}$ for the rest of the time. Then, we can correct for its effect on the PV signal. 


\subsection{Optical rotation detection}

In the polarimeter configuration chosen here, the imbalance is not only sensitive to linear dichroism relative to the $\hat{X}, \hat{Y}$ axes but also to an optical rotation 22. Consequently, a contribution to the atomic imbalance may arise from an atomic orientation in the $7 \mathrm{~S}$ state, $\mathcal{P}(\tau)=\operatorname{Tr}\left\{\rho_{e}(\tau) \vec{F} \cdot \hat{z}\right\}$. Strictly speaking it corresponds to an additional term in Eq. (23) (see 20]) which we have omitted here for two reasons: i) it vanishes in the ideal APV configuration; in real conditions, only combination of imperfections can lead to a nonzero signal; ii) instead of a linear dichroism effect, this corresponds to an optical rotation of the linear probe polarization, i.e. an optical effect which our method of measurement allows us to discriminate unambiguously against linear dichroism. The latter causes the probe polarization to rotate towards the axis of larger gain, while the sense of the rotation imposed by an optical activity is the same whatever the direction of the input polarization. Therefore, in order to distinguish between both effects, we use their opposite behavior when the direction of the probe polarization is switched in the vapor cell, with a half wave plate $(\lambda / 2)^{p r}$, from $\|$ to $\perp \hat{\epsilon}_{e x}$, configurations respectively dubbed para and ortho. By rotating the probe polarization through $90^{\circ}$ at cell entrance and by leaving the polarimeter and the excitation polarization unaltered, we switch the probe polarization from one bisector of the polarimeter eigenaxes to the other. Hence, the polarimeter still operates in balanced mode and the imbalance associated with the linear dichroism effect, once calibrated, remains identical in magnitude and sign. The effect of optical rotation on the other hand changes sign and can be rejected [22].

With this method, the probe polarization is rotated by $90^{\circ}$ with respect to the polarimeter axes. Another possibility consists in applying a second $90^{\circ}$ rotation to the probe polarization outgoing from the cell. Without tilts induced by the vapor, the probe polarization analyzed by the polarimeter in the ortho configuration recovers the same direction as that it has in the para one. This method a priori equivalent to the former one, has the advantage to make easy the use of a polarization magnifier, a dichroic component 25] which would otherwise come into conflict with measurements involving probe polarization rotations.

\subsection{Global rotation of the experiment about the beam axis}

As explained previously, the balanced-mode operation of the polarimeter, favoured for optimal detection of small asymmetries, requires that at the cell entrance we choose either a para or an ortho configuration. When we rotate $\hat{\epsilon}_{e x}$ by $45^{\circ}$ increments, we simultaneously rotate $\hat{\epsilon}_{p r}$ by the same angle. We do this for both the para and the ortho configurations. Thus, eight different pump-probe configurations (four para and four ortho) are used for the measurements. In principle, when we rotate $\hat{\epsilon}_{e x}$ we should rotate the polarimeter in order to ensure that its eigenaxes remain at $\pm 45^{\circ}$ angle from $\hat{\epsilon}_{e x}$, the axes of the main Stark alignment to which we want to remain insensitive. But this operation is hardly compatible with excellent mechanical stability. We have found that it can be advantageously replaced by a second $45^{\circ}$ rotation of $\hat{\epsilon}_{p r}$ at the cell exit, opposite to that performed at the entrance. All polarization rotations are performed by insertion and removal of half-wave plates placed on each input beam and before the polarimeter on the probe emerging from the cell. The output plates are oriented and controlled in a way which allows the analyzed probe polarization, in absence of tilts induced by the vapor, always to lie along the same bisector of the polarimeter eigen-axes, whatever the input configuration of the $\hat{\epsilon}_{e x}, \hat{\epsilon}_{p r}$ polarizations.

We can verify that this operation, where the cell is sandwiched between two half wave plates having their axes parallel, is physically equivalent to rotating the input polarization 
and the polarimeter by the same angle as that carried out by one half wave plate. Let us introduce the transformation $\mathrm{T}$ produced by the vapor cell whose effect on $\hat{\epsilon}_{p r}$ we want to measure and the transformation applied by insertion of a half wave plate $\mathcal{S}_{\delta}$, which is a symmetry with respect to the plate axes of direction $\delta$. With the plates absent, the polarizations at the input and output of the cell are: $\hat{\epsilon}_{\text {out }}=T \hat{\epsilon}_{\text {in }}$; with the input and output plates inserted, $\hat{\epsilon}_{i n}^{\prime}=\mathcal{S}_{\delta} \hat{\epsilon}_{i n}$ at cell entrance, while at entrance of the polarimeter $\hat{\epsilon}_{\text {out }}^{\prime}=\left(\mathcal{S}_{\delta} T \mathcal{S}_{\delta}\right) \hat{\epsilon}_{\text {in }}$. We note that the scalar product is conserved in the operation $\mathcal{S}_{\delta}$ and that $\left(\mathcal{S}_{\delta}\right)^{2}=\mathbb{I}$. The measured quantity in each channel is the square of the scalar product $\hat{X} \cdot \hat{\epsilon}_{\text {out }}^{\prime} \equiv \hat{X} \cdot\left(\mathcal{S}_{\delta} T \mathcal{S}_{\delta}\right) \hat{\epsilon}_{\text {in }} \equiv\left(\mathcal{S}_{\delta} \hat{X}\right) \cdot\left(T \mathcal{S}_{\delta} \hat{\epsilon}_{i n}\right)$. Hence, we measure the same quantity as if we had applied the same transformation to the polarimeter and to the input polarization.

To simplify the following discussion we suppose hereafter that the polarimeter is rotated in the same way as the probe polarization and that the axes $\hat{x}, \hat{y}, \hat{X}$ and $\hat{Y}$ are linked to the input polarizations and to the polarimeter in their global rotation about the beam axis. During the rotations, the two components of the probe polarization along the eigen-axes of the polarimeter, $\hat{\epsilon}_{X}, \hat{\epsilon}_{Y}$, remain at $\pm 45^{\circ}$ to the input probe polarization, both in para and ortho configurations.

To be complete, let us mention that we insert one more half-wave plate, $(\lambda / 2)^{\text {det }}$, just in front of the polarimeter which performs a symmetry of the outgoing probe polarization with respect to the symmetry plane of the polarimeter, for discrimination between true polarization tilts and instrumental imbalances [22]. Then, using the four reversals $\sigma_{c a l}=$ $\pm 1, \sigma_{E_{l}}= \pm 1, \sigma_{p r}= \pm 1, \sigma_{d e t}= \pm 1$, of the calibration angle $\theta_{c a l}$, the electric field $\vec{E}_{l}$ and half-wave plates $(\lambda / 2)^{p r}$ and $(\lambda / 2)^{\text {det }}$ respectively, the PV quantity $\theta^{p v}$ is reconstructed as the $E_{l}$-odd, calibrated linear dichroism:

$$
G=\theta_{c a l}\left\langle\sigma_{E_{l}}\left[\frac{<\sigma_{d e t} D^{a t}\left(\left\{\sigma_{j}\right\}\right)>_{\sigma_{d e t} \sigma_{c a l}}}{<\sigma_{d e t} \sigma_{c a l} D^{a t}\left(\left\{\sigma_{j}\right\}\right)>_{\sigma_{d e t} \sigma_{c a l}}}\right]\right\rangle_{\sigma_{E_{l}} \sigma_{p r}} .
$$

The experiment [11, 13] consists in recording successively the four values of $G_{y}, G_{x}, G_{Y}, G_{X}$ of $\mathrm{G}$ for the four configurations $\hat{y}, \hat{x}, \hat{Y}, \hat{X}$ of $\hat{\epsilon}_{e x}$. In the next sections of this paper, we show that the cylindrical symmetry can help in identifying and rejecting contributions to $G$ (Eq.(26) due to transverse $\vec{E}$ and $\vec{B}$ fields, or misalignments.

\subsection{Defect invariance under global rotations of the experiment}

We cannot take for granted that during global rotations of the experiment about the common beam axis all transverse fields remain invariant. In this section we want to discuss this assumption and present arguments which give it some support. There is no problem of course concerning the laboratory magnetic field imperfectly compensated, nor a possible transverse electric field which results from an $\hat{E}_{l} / \hat{k}$ misalignment and reverses with the voltage applied to the cell. But one may be concerned by the behavior of the electric and magnetic fields arising from the space charge developped inside the cesium cell. The space charge results from photoionization taking place on the cell windows at each excitation pulse [23]. Therefore, the question at issue here is that of a possible correlation between the direction of the excitation polarization and the electron distribution responsible for transverse $\vec{E}_{t}$ and $\vec{B}_{t}$ fields. Would such a correlation exist, our assumption would fail. Thus, we are concerned by the angular distribution of the electrons emitted by the surface of a sapphire crystal normal to the trigonal symmetry axis via the photoionization process. The linearly polarized photons, with $\lambda=540 \mathrm{~nm}$, propagate along the symmetry axis. In practice, the sapphire surface, at a temperature around $500 \mathrm{~K}$, constitutes the internal side of the windows of the cylindrical cell containing cesium vapor. (This latter is at saturated vapor 
pressure of a reservoir kept at $410 \mathrm{~K}$ ). Those windows have been annealed at a temperature of $\sim 1400 \mathrm{~K}$ before being mounted on the cylindrical tube. After annealing the surface is reconstructed: we have found by AFM imaging and laser beam diffraction 28 the presence of regularly spaced vicinal steps, several hundreds of nanometers apart. This situation is known to be favorable for reducing the adsorption probability, except for edge sites which favor chemisorption 29] but concern only a tiny fraction of the surface.

According to the findings of references [26] and [27, we shall assume that the surface states can be constructed mainly from the $\mathrm{Al} 3 p$ orbitals. We shall take for the density matrix describing the surface states the following expression:

$$
\rho_{S}=\sum_{i} a_{t}(i)\left(\left|3 p_{x}(i)\right\rangle\left\langle 3 p_{x}(i)|+| 3 p_{y}(i)\right\rangle\left\langle 3 p_{y}(i)\right|\right)+a_{l}(i)\left|3 p_{z}(i)\right\rangle\left\langle 3 p_{z}(i)\right|
$$

The summation $i$ runs over the surface aluminum atoms endowed with dangling $3 p$ bonds due to the presence of cesium adatoms. Under the experimental working conditions, $\mathrm{T}=500 \mathrm{~K}$, the surface coverage is small. It is then reasonable to assume that the dangling $\mathrm{Al} 3 p$ bonds are randomly distributed on the surface so that the phase factor between the orbitals $(i)$ and $(j), \exp \left(i\left(\vec{r}_{i}-\vec{r}_{j}\right) \cdot \vec{k}\right)$ is also randomly distributed. This explains why we have not included in $\rho_{S}$ non diagonal terms like $\left|3 p_{x}(i)\right\rangle\left\langle 3 p_{x}(j)\right|$.

Since the incident photons belong to the optical range it is legitimate to use the dipole approximation for describing the photoionization process. Centrifugal barrier effects imply that the $p \rightarrow s$ transition amplitude dominates the $p \rightarrow d$ one. Let us denote by $x \psi_{3 p}(r), y \psi_{3 p}(r)$ and $\psi_{s}^{\text {out }}\left(p_{e}, r\right)$ the wave functions associated respectively with the $3 p_{x}, 3 p_{y}$ orbitals and the outgoing $s$ wave electrons with momentum $p_{e}$. With the help of equation (27) the surface photoionization cross section is given, up to phase space factors, by:

$$
\sigma\left(3 p \rightarrow s, p_{e}\right) \propto\left|\int d^{3} r x \psi_{3 p}(r)\left(\vec{r} \cdot \vec{\epsilon}_{e x}\right) \psi_{s}^{\text {out }}\left(p_{e}, r\right)\right|^{2}+(x \rightarrow y)
$$

Remembering that the photon momentum is along the $z$ axis, we can write $\vec{r} \cdot \hat{\epsilon}_{e x}=$ $x \cos (\phi)+y \sin (\phi)$, where $\phi$ gives the direction of the polarization vector $\vec{\epsilon}_{e x}$ in the $x, y$ plane. Performing the space integral in equation (28) one sees that the first term in the right hand side is proportional to $\cos (\phi)^{2}$ and the second to $\sin (\phi)^{2}$ times the square of the same radial integral $\int_{0}^{\infty} d r r^{4} \psi_{3 p}(r) \psi_{s}^{\text {out }}\left(p_{e}, r\right)$. In more physical terms, it means that the distribution of the photo-electrons has no dependence on the direction of the photon linear polarization $\vec{\epsilon}_{e x}$ in the $x, y$ plane. Such a result hinges on the assumption that the surface states near the edges of the conduction band are produced mainly by $\mathrm{Al} 3 p_{x, y}$ orbitals, according to the authors of reference [26], section IV.A. It should be kept in mind that adding a contribution $\propto|3 s(i)\rangle\langle 3 s(i)|$ to the surface density matrix $\rho_{S}$ would indroduce a $\cos (\phi)^{2}$ dependence in the angular distribution of the photo-electrons ${ }^{1}$, like for instance in the photelectric effect in atomic hydrogen. No evidence for a $\cos (\phi)^{2}$ term has been found in the measured radial electric field produced by the beam of surface photo-electrons accelerated by an applied electric static field normal to the surface [23]. This gives some empirical support for the basic assumptions leading to formula (27), in particular, for the absence of $\mathrm{Al} 3 s$ orbital contributions.

\footnotetext{
${ }^{1}$ This can be verified by making in Eq. (28) the substitutions needed for the evaluation of $\sigma\left(3 s \rightarrow p, p_{e}\right)$ instead of $\sigma\left(3 p \rightarrow s, p_{e}\right)$, namely $x \psi_{3 p}(r) \rightarrow \psi_{3 s}(r)$ and $\psi_{s}^{\text {out }}\left(p_{e}, r\right) \rightarrow\left(\vec{p}_{e} \cdot \vec{r}\right) \psi_{p}^{\text {out }}\left(p_{e}, r\right)$ leading to an angular distribution of $\sigma\left(3 s \rightarrow p, p_{e}\right) \propto\left|\hat{\epsilon}_{e x} \cdot \vec{p}_{e}\right|^{2}$, hence to a $\cos (\phi)^{2}$ dependence in the transverse plane.
} 


\section{Cylindrical symmetry breaking generated by trans- verse $\vec{E}_{t}, \vec{B}_{t}$ fields or misalignment.}

In this section, we consider the modification of the the two-channel polarimeter imbalance $D^{a t}$ resulting from the simultaneous presence of stray transverse $\vec{E}_{t}, \vec{B}_{t}$ fields. The important feature of the present mechanism is that it can generate a PV-like signal which, although a physical scalar, shares the properties of the true PV signal and in particular the cylindrical symmetry. Fortunately, this potential source of systematic effect is accompanied by another imbalance contribution which breaks the cylindrical symmetry. In the present deviation from the ideal configuration, the imbalance signal anisotropy indicates the presence of possible systematic effect and can yield an upper bound for its magnitude.

\subsection{Alignment correction induced by $\vec{E}_{t}$ and $\vec{B}_{t}$ fields.}

Now, we evaluate the second-order correction to the alignment tensor $\Delta^{(2)} \mathcal{A}_{i j}^{e}$ generated by the combined action of the fields $\vec{E}_{t}$ and $\vec{B}_{t}$, using the method developed in section 2.2.1.

\subsection{1 $\vec{E}_{t}$-induced correction to the Stark alignment.}

We start by the first-order modification of the Stark alignment $\Delta^{(1)} \mathcal{A}_{i j}^{e}(0)$ resulting from the presence of $\vec{E}_{t}$. The vector $\vec{b}_{S t}$ which defines the Stark transition matrix has a correction term $\Delta_{E_{t}}^{(1)} \vec{b}=i \beta \hat{E}_{t} \wedge \hat{\epsilon}_{e x}$, which clearly lies along $\hat{k}$. The corresponding correction $\Delta^{(1)} \mathcal{A}_{i j}^{e}(0)$ is then proportional to $\left(\vec{b}_{S t}\right)_{i} \hat{k}_{j}+(i \leftrightarrow j)$. It does not contribute to the polarimeter imbalance $S_{X}-S_{Y} \propto \mathcal{D}_{i j} \cdot \mathcal{A}_{i j}^{e}$ since in the ideal configuration the detector tensor is purely transverse, i.e. $\hat{k}_{i} \cdot \mathcal{D}_{i j}=0$.

\subsection{2 $\quad \vec{B}_{t}$ Larmor precession of the $\vec{E}_{t}$ correction to the Stark alignment}

For there to be a non-zero correction, another kind of defect must be present. For instance a transverse magnetic field $\vec{B}_{t}$ would induce a Larmor precession of the tensor $\mathcal{A}_{i j}^{e}(0)$ (Eq. (12)) making the defect detectable by the polarimeter. The Larmor precession, of angular frequency $\omega_{F^{\prime}}$ is equivalent to a rotation of the vector $\vec{b}$ (and $\vec{b}^{*}$ ) about the axis $\hat{B}_{t}$ by the angle $\omega_{F^{\prime}} \tau=\gamma B_{t} \tau$. The duration $\tau$ represents the averaged time spent by the atoms in the excited state with typical values for short pump-probe delay between 5 and $15 \mathrm{~ns}$, depending mainly on the saturation. Note that the sign of the Larmor precession about $\vec{B}_{t}$, given here by the sign of $\omega_{F^{\prime}}$ is opposite in the two $7 S$ hyperfine states ${ }^{2}$. In practice $\left|\omega_{F^{\prime}}\right| \tau \ll 1$, so that the rotation can be considered as infinitesimal, and the variation of $\vec{b}$ induced by the Larmor precession can be written as: $\Delta_{L}^{(1)} \vec{b}=\gamma B_{t} \tau \hat{B}_{t} \wedge \vec{b}$. The combined effect of $\vec{E}_{t}$ and $\vec{B}_{t}$ is then described by a second-order correction to the vector $\vec{b}_{S t}$ :

$$
\Delta^{(2)} \overrightarrow{b_{S t}}=\Delta_{L}^{(1)}\left(\Delta_{E_{t}}^{(1)} \vec{b}_{S t}\right)=i \beta \gamma \tau \vec{B}_{t} \wedge\left(\vec{E}_{t} \wedge \hat{\epsilon}_{e x}\right)=i \beta \gamma \tau\left(\left(\vec{B}_{t} \cdot \hat{\epsilon}_{e x}\right) \vec{E}_{t}-\left(\vec{B}_{t} \cdot \vec{E}_{t}\right) \hat{\epsilon}_{e x}\right) .
$$

We obtain the mixed second order $\vec{E}_{t}, \vec{B}_{t}$ correction to $\mathcal{A}_{i j}^{e}(\tau)$ contributing to $S_{X}-S_{Y}$ by replacing in Eq. (12) $\operatorname{Re}\left\{b_{i} b_{j}^{*}\right\}$ by $\operatorname{Re}\left\{\left(\vec{b}_{S t}\right)_{i}\left(\Delta^{(2)} \vec{b}^{*}\right)_{j}+\left(\vec{b}_{S t}\right)_{j}\left(\Delta^{(2)} \vec{b}^{*}\right)_{i}\right\}:$

$\Delta^{(2)} \mathcal{A}_{i j}^{e}(\tau)=8 g_{F^{\prime}}^{2} \beta^{2} E_{l} \gamma \tau \mathcal{F}\left(\left(\vec{B}_{t} \cdot \vec{E}_{t}\right)\left(\hat{E}_{l} \wedge \hat{\epsilon}_{e x}\right)_{i}\left(\hat{\epsilon}_{e x}\right)_{j}-\left(\vec{B}_{t} \cdot \hat{\epsilon}_{e x}\right)\left(\hat{E}_{l} \wedge \hat{\epsilon}_{e x}\right)_{i}\left(\vec{E}_{t}\right)_{j}+(i \leftrightarrow j)\right)$.

\footnotetext{
${ }^{2}$ Given that the magnetic moment of the electron is negative, the precession occurs in the positive sense for the $F^{\prime}=I+1 / 2$ sublevel with $g_{F^{\prime}}>0$.
} 
Note that the first term in the above expression of $\Delta^{(2)} \mathcal{A}_{i j}^{e}$ is proportional to $\Delta_{p v} \mathcal{A}_{i j}^{e}$ (see Eq.(15) ) and, as a consequence, is expected to lead to a contribution to $S_{X}-S_{Y}$ simulating the true PV contribution, if $\vec{E}_{t} \cdot \vec{B}_{t}$ is even under the $\vec{E}_{l}$ reversal.

To get the final correction to the atomic imbalance $D^{a t}$ we perform the tensor contraction of $\Delta^{(2)} \mathcal{A}_{i j}^{e}(\tau)$ with the differential detector tensor $\mathcal{D}_{i j}$ :

$$
\begin{aligned}
\Delta^{(2)} D_{a t}\left(\vec{E}_{t}, \vec{B}_{t}\right)=K \Delta^{(2)} \mathcal{A}_{i j}^{e}(\tau) \cdot \mathcal{D}_{i j}=8 g_{F^{\prime}}^{2} \beta^{2} E_{l} \gamma \tau K \mathcal{F} \times \\
\\
{\left[\left(\left(\vec{B}_{t} \cdot \vec{E}_{t}\right) \mathcal{S}_{c h i r}\left(\hat{\epsilon}_{X}, \hat{E}_{l}\right)-\left(\vec{B}_{t} \cdot \hat{\epsilon}_{e x}\right)\left(\hat{E}_{l} \wedge \hat{\epsilon}_{e x} \cdot \hat{\epsilon}_{X}\right)\left(\vec{E}_{t} \cdot \hat{\epsilon}_{X}\right)\right)-(X \leftrightarrow Y)\right], }
\end{aligned}
$$

where $\mathcal{S}_{\text {chir }}\left(\hat{\epsilon}_{X}, \hat{E}_{l}\right)=-\mathcal{S}_{\text {chir }}\left(\hat{\epsilon}_{Y}, \hat{E}_{l}\right)$ is the pseudo-scalar given by the formula (22). It is quite clear, as announced, that the first term, although being a physical scalar, due to the pseudo-scalar T-even factor $\gamma \tau\left(\hat{B}_{t} \cdot \hat{E}_{t}\right)$ is a potential source of sytematic error for the PV signal. In contrast to the PV-like term, the second term contains an anisotropic part. It is convenient to rewrite $D^{a t}$ under a form where $\mathcal{S}_{\text {chir }}\left(\hat{\epsilon}_{X}, \hat{E}_{l}\right)$ appears as an overall factor. To do this, we use the formulae (20) defining $\hat{\epsilon}_{X}$ and $\hat{\epsilon}_{Y}$ and the explicit form of $\mathcal{S}_{\text {chir }}\left(\hat{\epsilon}_{X}, \hat{E}_{l}\right)$ given in Eq. (22). Performing explicitely the antisymmetrization with respect to the exchange $X \leftrightarrow Y$, one arrives at the expression:

$$
\Delta^{(2)} D^{a t}\left(\vec{E}_{t}, \vec{B}_{t}\right)=16 g_{F^{\prime}}^{2} \beta^{2} E_{l} \gamma \tau K \mathcal{F} \mathcal{S}_{c h i r}\left(\hat{\epsilon}_{X}, \hat{E}_{l}\right)\left(\vec{B}_{t} \cdot \vec{E}_{t}-\left(\vec{B}_{t} \cdot \hat{\epsilon}_{e x}\right)\left(\vec{E}_{t} \cdot \hat{\epsilon}_{e x}\right)\right),
$$

which exhibits the presence of a signal anisotropy under the global rotation of the experiment. Note that the last parenthesis can be split into the sum of a purely isotropic contribution and a purely anisotropic one:

$$
\frac{1}{2} \vec{B}_{t} \cdot \vec{E}_{t}-\left(\left(\vec{B}_{t} \cdot \hat{\epsilon}_{e x}\right)\left(\vec{E}_{t} \cdot \hat{\epsilon}_{e x}\right)-\frac{1}{2} \overrightarrow{B_{t}} \cdot \overrightarrow{E_{t}}\right) .
$$

We can write explicit expressions for the variations of this geometrical factor when global rotations of the experiment are performed. The initial position of $\hat{\epsilon}_{e x} \| \hat{y}$ in the laboratory frame will serve as a reference axis. If we define the angles: $\left(\hat{y}, \hat{\epsilon}_{e x}\right)=\phi,\left(\hat{y}, \hat{E}_{t}\right)=\theta_{E_{t}}$ and $\left(\hat{y}, \hat{B}_{t}\right)=\theta_{B_{t}}$, we obtain:

$$
\left(\hat{B}_{t} \cdot \hat{E}_{t}-\left(\hat{B}_{t} \cdot \hat{\epsilon}_{e x}\right)\left(\hat{E}_{t} \cdot \hat{\epsilon}_{e x}\right)\right)=\frac{1}{2}\left(\cos \left(\theta_{B_{t}}-\theta_{E_{t}}\right)-\cos \left(2 \phi-\theta_{B_{t}}-\theta_{E_{t}}\right)\right) .
$$

We note that this expression is independent of the direction chosen as origin of angular coordinates, as it should be. Furthermore, the purely anisotropic contribution has a dependence on the rotation angle $\phi$ which only involves Fourier components at the frequency $2 \phi$. Consequently, four measurements corresponding to $\hat{\epsilon}_{e x} \| \hat{y}, \hat{X}, \hat{x}$, and $\hat{Y}$ are sufficient for us to obtain full information about the effect induced by the spurious fields $\vec{E}_{t}$, and $\vec{B}_{t}$. We notice that $\Delta^{(2)} D^{a t}\left(\vec{E}_{t}, \vec{B}_{t}\right)$ cancels when $\vec{E}_{t}$ is parallel to $\hat{\epsilon}_{e x}$, as expected.

This atomic imbalance, like the PV one, is to be compared in magnitude to that of the calibration tilt angle (Eq. 24):

$$
\begin{aligned}
\theta_{c a l} \frac{\Delta^{(2)} D^{a t}\left(\vec{E}_{t}, \vec{B}_{t}\right)}{D^{a t}\left(\theta_{c a l}\right)} & =G_{E B}\left(\cos \left(2 \phi-\theta_{B_{t}}-\theta_{E_{t}}\right)-\cos \left(\theta_{B_{t}}-\theta_{E_{t}}\right)\right) \\
G_{E B} & =\omega_{F^{\prime}} \tau \frac{E_{t}}{E_{l}}\left(\hat{z} \cdot \hat{E}_{l}\right)=\gamma B_{t} \tau \frac{E_{t}}{E_{l}}\left(\hat{z} \cdot \hat{E}_{l}\right) .
\end{aligned}
$$

where 
Like the PV one (Eq. 225), this ratio is odd under $\vec{E}_{l}$ reversal, if the quantity $\vec{E}_{t} \cdot \vec{B}_{t}$ is even. Therefore, we have shown the possible existence of a magnetoelectric optical effect which, in a given $\left(\hat{\epsilon}_{e x}, \hat{\epsilon}_{p r}\right)$ configuration, can contribute to the APV quantity, the $E_{l}$-odd, calibrated linear dichroism $G$, defined by Eq. (26).

\subsection{Properties of the isotropic and anisotropic contributions}

The signal corresponding to expression (31) has the symmetry properties expected for a signal of electromagnetic origin. It is invariant under space reflexion. The isotropic contribution only differs from the APV signal by the pseudoscalar factor $\vec{E}_{t} \cdot \vec{B}_{t}$ which cancels out only if the two transverse fields are orthogonal. It is also invariant under time reversal since the precession angle $\omega \tau$ and $\vec{B}_{t}$ are both T-odd quantities. This isotropic contribution mimics the APV signal only if its behaviour in $E_{l}$-field reversal, like that of the APV signal is odd, that is to say if $\left(\vec{E}_{t} \cdot \vec{B}_{t}\right)$ is itself even under this reversal: i.e. both fields are even or both odd. Hence there are two different contributions. When we perform successive simultaneous rotations of $\hat{\epsilon}_{e x}$ and $\hat{\epsilon}_{p r}$ by increments of $45^{\circ}, \hat{\epsilon}_{e x}=\hat{y}, \hat{X}, \hat{x}, \hat{Y}\left(\phi=0^{\circ}, 45^{\circ}, 90^{\circ}, 135^{\circ}\right)$, the transverse fields remain fixed. Measuring the four calibrated linear dichroism signals $G_{y}, G_{Y}, G_{x}, G_{X}$, actually provides us with two independent evaluations of the isotropic part:

$$
\begin{aligned}
\mathrm{S}_{\mathrm{xy}} & =\frac{1}{2}\left(G_{x}+G_{y}\right)=\theta^{p v}+\overline{G\left(\vec{E}_{t}, \vec{B}_{t}\right)} \\
\mathrm{S}_{\mathrm{XY}} & =\frac{1}{2}\left(G_{X}+G_{Y}\right)=\theta^{p v}+\overline{G\left(\vec{E}_{t}, \vec{B}_{t}\right)}
\end{aligned}
$$

where

$$
\overline{G\left(\vec{E}_{t}, \vec{B}_{t}\right)}=-G_{E B}^{+} \cos \left(\theta_{B_{t}^{+}}-\theta_{E_{t}^{+}}\right)-G_{E B}^{-} \cos \left(\theta_{B_{t}^{-}}-\theta_{E_{t}^{-}}\right) .
$$

We have used the + and - superscripts to distinguish the case where $\vec{E}_{t}$ and $\vec{B}_{t}$ are both even or both odd in $\vec{E}_{l}$ reversal. The important point is that we expect - and we observe [11] - equality to within noise between $\mathrm{S}_{\mathrm{xy}}$ and $\mathrm{S}_{\mathrm{XY}}{ }^{3}$. On the other hand, the signal anisotropy can be evaluated from the differences $\mathrm{D}_{\mathrm{xy}} \equiv \frac{1}{2}\left(G_{x}-G_{y}\right)$ and $\mathrm{D}_{\mathrm{XY}} \equiv \frac{1}{2}\left(G_{X}-G_{Y}\right)$ :

$$
\begin{aligned}
\mathrm{D}_{\mathrm{xy}} & =-G_{E B}^{+} \cos \left(\theta_{E_{t}^{+}}+\theta_{B_{t}^{+}}\right)-G_{E B}^{-} \cos \left(\theta_{E_{t}^{-}}+\theta_{B_{t}^{-}}\right), \\
\mathrm{D}_{\mathrm{XY}} & =-G_{E B}^{+} \sin \left(\theta_{E_{t}^{+}}+\theta_{B_{t}^{+}}\right)-G_{E B}^{-} \sin \left(\theta_{E_{t}^{-}}+\theta_{B_{t}^{-}}\right) .
\end{aligned}
$$

In order to obtain $\overline{G\left(\vec{E}_{t}, \vec{B}_{t}\right)}$ we need additional information we can actually extract from complementary measurements performed in presence of an auxiliary magnetic field. Measurements similar to the PV ones made in such a field of known direction and magnitude ( $2 \mathrm{G}$ ) yield the directions and magnitudes of $\vec{E}_{t}^{+}$and $\vec{E}_{t}^{-}$, the transverse field contributions even and odd in $\vec{E}_{l}$ reversal. However, as we shall see in the section 5 , the situation is somewhat complicated by the presence of symmetry breaking effects of another kind which lead to purely anisotropic contributions.

\footnotetext{
${ }^{3}$ Let us note that in the case where $\vec{E}_{t}$ would exhibit a $\cos ^{2}(\phi)$ modulation, though considered unlikely (§ 3.6) then the linear dichroism signals $G_{y}, G_{Y}, G_{x}, G_{X}$ would acquire a $\cos (4 \phi)$ modulation. It can be predicted that this would lead to a difference between $S_{x y}$ and $S_{X Y}$, which is not observed in our measurements. $\mathrm{D}_{\mathrm{xy}}$ and $\mathrm{D}_{\mathrm{XY}}$ would not be modified.
} 


\subsection{Comment on the $\vec{E}_{t} \cdot \vec{B}_{t}$ induced magnetoelectric dichroism}

We have just demonstrated theoretically the existence in our atomic system of a linear dichroism generated by two parallel, transverse fields $\vec{E}_{t}$ and $\vec{B}_{t}$. Its magnitude is proportional to $\vec{E}_{t} \cdot \vec{B}_{t}$ and its axes are oriented at $\pm 45^{\circ}$ to the field direction. Our observations confirm the expected magnitude (see $\S .6$ for a more detailed discussion). We have actually exploited it thoroughly for controlling the stray $E_{t}$-fields generated in Cs filled alumina cells by electric charges produced by photoionization [23].

Such an effect supported by symmetry arguments was predicted long ago by Jones 30 . Despite searches in molecular liquids, it remained unobserved until recently [31, when the samples were placed under extreme experimental conditions: a $B_{t}$ field of $15 \mathrm{~T}$ and an $E_{t}$ field of $1.75 \mathrm{kV} / \mathrm{cm}$. Therefore it is worth underlining the differences between our present experimental conditions and those which correspond to the molecular liquid samples used for this kind of observation: number densities about $10^{6}$ times lower, magnetic fields $10^{4}$ to $10^{7}$ times smaller, and a transverse $\vec{E}_{t}$ field about one hundredth of the size of the longitudinal $\vec{E}_{l}$ field of comparable magnitude which provides the vapor with axial symmetry. Above all one should bear in mind that in the present case we have a dilute atomic sample in a particularly simple atomic state. Consequently, the effect of the magnetoelectric dichroism observed on the probe beam can be given a simple, intuitive and quantitative interpretation: the Larmor precession of the atomic alignment generated in the excited state by interference between the Stark-induced dipoles involving both the longitudinal and transverse components of the electric field. But this mechanism is not unique. For other illustrations which lend themselves to detailed calculations also in the context of atomic vapors excited via a highly forbidden transition, see for instance the forthcoming section 4.5 and reference 32 .

\subsection{Combined effect of an $\vec{E}_{t}$ field and a pump-probe misalignment}

We have shown in subsection 4.1 that the alignment associated with a small transverse electric field $\vec{E}_{t}$ leads to an atomic imbalance in presence of a transverse magnetic field $\vec{B}_{t}$. (The case of an $E_{l}$-odd $\vec{E}_{t}$ field accounts for a misalignment between the applied $\vec{E}_{l}$ field and the excitation beam direction $\hat{k}_{e x}$ ). We now want to show that a misalignement of the probe beam with respect to the excitation beam leads to the same effect. This deviation from the ideal configuration can be described by applying to the polarized probe beam an infinitesimal rotation by a small angle $\delta \alpha$ about a unit vector $\hat{n}$ normal to the excitation photon momentum $\vec{k}_{e x}$ such that $\delta \alpha \hat{n}=\hat{k}_{e x} \wedge \hat{k}_{p r}$.

Such an infinitesimal rotation of the probe beam leads to a correction $\Delta_{m i s}^{(1)} \mathcal{D}_{i j}$ of the detection tensor (Eq. (17)) which is given by:

$$
\Delta_{m i s}^{(1)} \mathcal{D}_{i j}=\delta \alpha\left(\left(\hat{n} \wedge \hat{\epsilon}_{X}\right)_{i}\left(\hat{\epsilon}_{X}\right)_{j}+\left(\hat{\epsilon}_{X}\right)_{i}\left(\hat{n} \wedge \hat{\epsilon}_{X}\right)_{j}-(X \rightarrow Y)\right) .
$$

By combining the effect of the probe beam tilt with the transverse electric field $\hat{E}_{t}$ correction

to the alignment tensor $\Delta^{(1)} \mathcal{A}_{i j}^{e} \propto b_{i} \Delta_{E_{t}}^{(1)} b_{j}+(i \leftrightarrow j)$, we predict a second-order correction to the atomic imbalance $D^{a t}$ :

$$
\Delta^{(2)} D^{a t}\left(E_{t}, m i s\right)=K \Delta^{(1)} \mathcal{A}_{i j}^{e}(0) \cdot \Delta_{m i s}^{(1)} \mathcal{D}_{i j} .
$$

Performing explicitely the tensor contraction together with the antisymetrisation with respect to $X \leftrightarrow Y$, we obtain the final expressions:

$$
\Delta^{(2)} D^{a t}\left(E_{t}, m i s\right)=-16 g_{F^{\prime}}^{2} \beta^{2} E_{l} \delta \alpha E_{t} K \mathcal{F} \mathcal{S}_{c h i r}\left(\hat{\epsilon}_{X}, \hat{E}_{l}\right)\left(\hat{n} \cdot \hat{E}_{t}-\left(\hat{n} \cdot \hat{\epsilon}_{e x}\right)\left(\hat{E}_{t} \cdot \hat{\epsilon}_{e x}\right)\right),
$$


$\theta_{c a l} \frac{\Delta^{(2)} D^{a t}\left(E_{t}, m i s\right)}{D^{a t}\left(\theta_{c a l}\right)}=G_{E \delta \alpha}\left(\hat{n} \cdot \hat{E}_{t}-\left(\hat{n} \cdot \hat{\epsilon}_{e x}\right)\left(\hat{E}_{t} \cdot \hat{\epsilon}_{e x}\right)\right)$, with $G_{E \delta \alpha}=\delta \alpha \frac{E_{t}}{E_{l}}\left(\hat{z} \cdot \hat{E}_{l}\right)$.

Thus only an $\vec{E}_{l}$-even transverse field, $\vec{E}_{t}^{+}$, can give rise to a harmful $\vec{E}_{l}$-odd imbalance. It is also interesting to note the close similarity of the above result with that induced by $E_{t}, B_{t}$ transverse fields Eq. (31) in $\S 4.1$, with just a single substitution performed on the P-even T-even rotational invariant:

$$
\omega_{F^{\prime}} \hat{B}_{t} \longrightarrow-\delta \alpha \hat{n}
$$

It is actually not surprising that the Larmor precession and a small rotation of the probe polarized beam lead to similar effects. Both effects arise from the same correction to the second-rank tensor $\Delta^{(1)} \mathcal{A}_{i, j}^{e}$, via either a small rotation of this tensor itself about $\hat{B}_{t}$ in the former case, or a small rotation of the detection tensor about $\hat{n}$ in the latter case. The above exact correspondance, and in particular the change of sign, can be understood from rather simple rotational invariance considerations ${ }^{4}$.

\subsection{Other mechanisms involving the $M_{1}$ amplitude}

As already noted in $\S 2.2 .4$, for there to be a systematic effect involving the $M_{1}$ amplitude there must also be a a transverse electric field. We also stressed that such a field alone is not enough. We now show how combining both electric and magnetic transverse fields can generate a systematic effect. Since the typical value of $\left|M_{1} / \beta E_{l}\right|$ in our experiments is $\simeq 2 \times 10^{-2}$, it looks like we could consider an $M_{1}$-Stark-induced contribution as a third order effect. In reality, because the presence of a $\vec{B}_{t}$ field makes slight mixing of the different hyperfine states, the $M_{1}$-Stark interference effect is enhanced. In fact, given that the hyperfine substates are no longer pure $\mathrm{F}$ states, the scalar Stark amplitude $\alpha \vec{E}_{l} \cdot \hat{\epsilon}_{e x}$ can connect S-states having different hyperfine quantum numbers. As a result, the size of the perturbation is reinforced by a factor $|\alpha / \beta| \simeq 10$. This is why we now want to focus our discussion on this particular contribution.

The matrix element of the scalar Stark-induced amplitude between the $6 S, F$ and the $7 S, F^{\prime}$ states with $F^{\prime} \neq F$ is calculated using first order perturbation theory ${ }^{5}$. It is convenient to write the result in terms of a correction contribution to the effective dipole $\vec{d}^{e f f}$ (see

\footnotetext{
${ }^{4}$ Let us first illustrate this point for a simpler configuration: $\vec{a}$ and $\vec{b}$ are assumed to be two physical 3-dimensional vectors and $R(\hat{n}, \phi)$ a rotation acting only upon the vector $\vec{b}$. Let us assume further that the scalar product $\vec{a} \cdot(R(\hat{n}, \phi) \vec{b})$ is associated with some physical observable; it is invariant under any global rotation $R^{\prime}$ acting on the two vectors $\vec{a}$ and $\vec{b}$. By choosing $R^{\prime}$ to be the inverse of $R$ : $R^{\prime}=R^{-1}(\hat{n}, \phi)=$ $R(\hat{n},-\phi)$, we get immediately the identity:

$$
\vec{a} \cdot(R(\hat{n}, \phi) \vec{b})=R^{\prime} \vec{a} \cdot\left(R^{\prime} R(\hat{n}, \phi) \vec{b}\right)=\left(R^{-1}(\hat{n}, \phi) \vec{a}\right) \cdot \vec{b} .
$$

The physical content of the above equation can be stated as follows: For a physical measurement involving the scalar product of two vectors, the effect of a rotation $R$ acting upon the vector on the r.h.s. is identical to that of the inverse rotation $R^{-1}$ acting upon the vector on the l.h.s..

What is involved here is actually the same idendity, but applied to the contraction of two second-order tensors, $A_{i j} \cdot B_{i j}$. It is not difficult to generalize. We can begin by considering the case of two factorized tensors: $A_{i j}=a_{i} b_{j}$ and $B_{i j}=c_{i} d_{j}$. The contraction of them reads: $A_{i j} \cdot B_{i j}=(\vec{a} \cdot \vec{c})(\vec{b} \cdot \vec{d})$. Let us rotate the r.h.s tensor: $B_{i j} \rightarrow B_{i j}^{R}=(R \vec{c})_{i}(R \vec{d})_{j}$. We get immediately: $A_{i j} \cdot B_{i j}^{R}=(\vec{a} \cdot R \vec{c})(\vec{b} \cdot R \vec{d})=$ $\left(R^{-1} \vec{a} \cdot \vec{c}\right)\left(R^{-1} \vec{b} \cdot \vec{d}\right)=A_{i j}^{R^{-1}} \cdot B_{i j}$. Then, the property can be extended to the general case by noting that an arbitrary second-order tensor $A_{i j}$ can always be written as a linear combination of the nine factorized tensors constructed from three independent vectors: $\vec{a}_{1}, \vec{a}_{2}, \vec{a}_{3}$.

${ }^{5}$ We deliberately omit here the magnetic perturbation of the $6 S, F$ state which has a larger hyperfine structure and leads to an effect about 5 times smaller.
} 
Eq. (1)):

$$
\Delta_{\alpha E_{t}, B}^{(2)} \vec{d}^{e f f}=-\alpha \vec{E}_{t}\left(F^{\prime}-F\right) \frac{\gamma_{S} B}{\Delta W_{7 S}} P_{F^{\prime}} \vec{\sigma} \cdot \hat{B} P_{F},
$$

where $\gamma_{S}\left(=\gamma / g_{F}\right)$ is the gyromagnetic factor for the electron spin. We write the resulting correction to the complex vector $\vec{b}$ which appears in the effective transition matrix $T_{\text {eff }}$ (see Eq. (5)) as:

$$
\Delta_{\alpha E_{t}, B}^{(2)} \vec{b}=\alpha\left(\vec{E}_{t} \cdot \hat{\epsilon}_{e x}\right)\left(F^{\prime}-F\right) \frac{\gamma_{S} B_{t}}{\Delta W_{7 S}} P_{F^{\prime}} \hat{B}_{t} P_{F} .
$$

We insert the above result in the general expression of the excited state alignment, Eq. (13), to get a new $M_{1}$ amplitude contribution $\propto \operatorname{Re}\left\{\left(b_{M_{1}}\right)_{i} \Delta^{(2)} b_{j}+(i \leftrightarrow j)\right\}$, where $\vec{b}_{M_{1}}=$ $-M_{1} \hat{k} \wedge \hat{\epsilon}_{e x}$. We now have all we need to write the alignment of the excited state resulting from the interference between the $M_{1}$ and the hyperfine-mixing scalar Stark amplitudes:

$$
\Delta_{\left(M_{1} \alpha E_{t}, B\right)}^{(3)} \mathcal{A}_{i j}=4 g_{F^{\prime}}^{2} \mathcal{F}\left(F^{\prime}-F\right) \frac{\gamma_{S} B}{\Delta W_{7 S}}\left(M_{1} \alpha \vec{E}_{t} \cdot \hat{\epsilon}_{e x}\right)\left(\left(\hat{B}_{t}\right)_{i}\left(\hat{k} \wedge \hat{\epsilon}_{e x}\right)_{j}+(i \leftrightarrow j)\right) .
$$

Contracting this tensor with the detection tensor $\mathcal{D}_{i j}$ we get the following contribution to the atomic imbalance:

$$
\begin{aligned}
\Delta^{(3)} D^{a t}\left(M_{1}, \alpha E_{t}, B_{t}\right)= & 16 g_{F^{\prime}}^{2} \mathcal{F} K\left(F^{\prime}-F\right) \frac{\gamma_{S}}{\Delta W_{7 S}} M_{1} \alpha \mathcal{S}_{c h i r}\left(\hat{\epsilon}_{X}, \hat{E}_{l}\right)\left(\vec{E}_{t} \cdot \hat{\epsilon}_{e x}\right)\left(\vec{B}_{t} \cdot \hat{\epsilon}_{e x}\right)\left(\hat{k} \cdot \hat{E}_{l}\right) \\
= & 16 g_{F^{\prime}}^{2} \mathcal{F} K\left(F^{\prime}-F\right) \frac{\gamma_{S}}{\Delta W_{7 S}} M_{1} \alpha \mathcal{S}_{c h i r}\left(\hat{\epsilon}_{X}, \hat{E}_{l}\right)\left(\hat{k} \cdot \hat{E}_{l}\right) \times \\
& {\left[\frac{1}{2} \vec{E}_{t} \cdot \vec{B}_{t}+\left(\left(\vec{B}_{t} \cdot \hat{\epsilon}_{e x}\right)\left(\vec{E}_{t} \cdot \hat{\epsilon}_{e x}\right)-\frac{1}{2} \vec{E}_{t} \cdot \vec{B}_{t}\right)\right] }
\end{aligned}
$$

The last factor in the r.h.s of the above equation has been split into its isotropic and its purely anisotropic parts. It is interesting to compare it with that obtained previously for the $\vec{E}_{t} \cdot \vec{B}_{t}$ effect, Eq. (32): the only difference lies in a reversal of the relative sign of these two contributions. To satisfy the $\vec{E}_{l}$-odd behavior, here, in contrast to the previous case, the scalar product $\vec{E}_{t} \cdot \vec{B}_{t}$ has to be odd under $\vec{E}_{l}$ reversal. This is why it has been considered here, even though it would be about 20 times smaller were the $\vec{E}_{l}$-odd and $\vec{E}_{l}$-even contributions to $\vec{E}_{t} \cdot \vec{B}_{t}$ of equal magnitude.

One may note also the presence of the additional factor $\left(\hat{k} \cdot \hat{E}_{l}\right)$ in the above expression (42): it has appeared in a natural way via the contribution of the $M_{1}$ amplitude and, combined with the factor $\left(\vec{B}_{t} \cdot \hat{\epsilon}_{e x}\right)$, it ensures T-reversal invariance of the result in which, by contrast to the Larmor precession dependent contribution (Eq. (31)), the time no longer appears explicitly.

Although we are not going to present here the details of the corresponding calculation, we would like to mention that the atomic imbalance associated to the vector Stark amplitude, instead of the scalar one, involves this time the same angular dependence as that given by Eq. (32). Note that this contribution is suppressed by a factor $|\beta / \alpha|=1 / 10$ with respect to that given by Eq. (42).

For completeness, let us quote the result deduced from Eq.(42) after normalization by the calibration imbalance (Eq. (24)):

$$
\theta_{c a l} \frac{\Delta^{(3)} D^{a t}\left(M_{1}, E_{t}, B_{t}\right)}{D^{a t}\left(\theta_{c a l}\right)}=-\left(F^{\prime}-F\right) \frac{\gamma_{S} B_{t}}{\Delta W_{7 S}} \frac{M_{1} \alpha E_{t}}{\left(\beta E_{l}\right)^{2}}\left(\hat{E}_{t} \cdot \hat{\epsilon}_{e x}\right)\left(\hat{B}_{t} \cdot \hat{\epsilon}_{e x}\right)\left(\hat{k} \cdot \hat{E}_{l}\right) .
$$




\section{Symmetry breaking by two transverse magnetic fields, $\vec{E}_{l}$-odd and $\vec{E}_{l}$-even}

Let us suppose that two transverse magnetic fields, $\vec{B}_{t}^{+}$and $\vec{B}_{t}^{-}$, one even and the other odd under $\vec{E}_{l}$-reversal are now the source of cylindrical symmetry breaking. Since the dominant Stark alignment is $\vec{E}_{l}$-even, those two fields having opposite behavior under $\vec{E}_{l}$-reversal can introduce an $\vec{E}_{l}$-odd character in the effect arising from perturbation to second order. Different possible mechanisms can enter into play, so we shall first consider one of them in detail. Afterwards, we shall present a generalization of the main features of the results.

\subsection{Larmor precession of the Stark alignment about a $\vec{B}_{t}$ field chang- ing its direction under $\vec{E}_{l}$ reversal}

We consider first the effect of Larmor precession. We shall show that the dominant Stark alignment (Eq. (14)) precessing in a transverse magnetic field acquires a detectable component of second-order in the infinitesimal precession angle $\omega_{F^{\prime}} \tau=\gamma \tau B_{t}$. The variation of $\vec{b}_{S t}$ induced by the Larmor precession can be written:

$$
\Delta_{L}^{(1)} \vec{b}_{S t}=i \beta \gamma \tau \vec{B}_{t} \wedge\left(\vec{E}_{l} \wedge \hat{\epsilon}_{e x}\right)=i \beta \omega_{F^{\prime}} \tau\left(\left(\hat{B}_{t} \cdot \hat{\epsilon}_{e x}\right) \vec{E}_{l}-\left(\hat{B}_{t} \cdot \vec{E}_{l}\right) \hat{\epsilon}_{e x}\right) .
$$

Since $\vec{B}_{t} \cdot \vec{E}_{l}=0$, the second term cancels, and since $\vec{E}_{l}$ is along $\hat{k}_{p r}$, it can be easily verified that the second order correction to the alignment of the type $\left(\Delta_{L}^{(1)} \vec{b}\right)_{i}\left(\Delta_{L}^{(1)} \vec{b}^{*}\right)_{j}+i \leftrightarrow j$ cannot contribute to a polarimeter imbalance. Even so, a possible misalignment between the pump and the probe beams combining to the first order Larmor correction can contribute, a situation that will be considered later on $(\S 5.3)$.

For the moment, we calculate the second-order correction to $\vec{b}$ associated with an infinitesimal rotation angle $\phi=\omega_{F^{\prime}} \tau$ about $\hat{B}_{t}$ to second order in $\phi$. We can separate $\vec{b}$ into its components parallel and perpendicular to the rotation axis:

$$
\vec{b}=\vec{b}_{\|}+\vec{b}_{\perp}=\left(\vec{b} \cdot \hat{B}_{t}\right) \hat{B}_{t}+\left(\vec{b}-\left(\vec{b} \cdot \hat{B}_{t}\right) \hat{B}_{t}\right) .
$$

The transformed vector is then written:

$$
\mathcal{R} \vec{b}=\vec{b}_{\|}+\vec{b}_{\perp} \cos \phi+\hat{B}_{t} \wedge \vec{b}_{\perp} \sin \phi,
$$

which we expand to second order in $\phi$ :

$$
\begin{aligned}
\mathcal{R} \vec{b} & =\vec{b}_{\|}+\vec{b}_{\perp}\left(1-\frac{\phi^{2}}{2}\right)+\left(\hat{B}_{t} \wedge \vec{b}_{\perp}\right) \phi+\mathcal{O}\left(\phi^{3}\right) \\
& =\vec{b}+\left(\hat{B}_{t} \wedge \vec{b}\right) \phi+\left(\left(\vec{b} \cdot \hat{B}_{t}\right) \hat{B}_{t}-\vec{b}\right) \frac{\phi^{2}}{2}+\mathcal{O} \phi^{3} .
\end{aligned}
$$

In the present situation, we are only interested in the contribution which alters the direction of $\vec{b}_{S t}$ along $\hat{h}=\hat{E}_{l} \wedge \hat{\epsilon}_{e x}$ at $\tau=0$ :

$$
\Delta_{L}^{(2)} \vec{b}_{S t}=i \beta \frac{\omega_{F^{\prime}}^{2} \tau^{2}}{2}\left(\hat{E}_{l} \wedge \hat{\epsilon}_{e x} \cdot \hat{B}_{t}\right) \hat{B}_{t} .
$$

This gives rise to a second order correction to $\mathcal{A}_{i j}^{S t}(\tau)$ obtained by replacing in Eq. (14) $\operatorname{Re}\left\{b_{i} b_{j}^{*}\right\}$ by $\left(\left(\vec{b}_{S t}\right)_{i}\left(\Delta_{L}^{2} \vec{b}_{S t}\right)_{j}+i \leftrightarrow j\right)$, which leads to the detectable alignment :

$$
\Delta_{L}^{(2)} \mathcal{A}_{i j}^{e}(\tau)=2 g_{F^{\prime}}^{2} \beta^{2} E_{l} \omega_{F^{\prime}}^{2} \tau^{2} \mathcal{F}\left(\hat{h} \cdot \hat{B}_{t}\right)\left(\hat{h}_{i}\left(\hat{B}_{t}\right)_{j}+i \leftrightarrow j\right) .
$$


After contraction with the second-rank detection tensor, the last factor in parentheses can be written $4 \mathcal{S}_{\text {chir }}\left(\hat{\epsilon}_{X}, \hat{E}_{l}\right)\left(\vec{B}_{t} \cdot \hat{\epsilon}_{e x}\right)$. This imbalance would not be $\vec{E}_{l}$-odd unless $\vec{B}_{t}$ were the sum of two fields having opposite behaviour under $\vec{E}_{l}$ reversal $\left(\vec{B}_{t}=\vec{B}_{t}^{+}+\vec{B}_{t}^{-}\right)$, which means that $\vec{B}_{t}$ would change direction when $\vec{E}_{l}$ is reversed. We arrive at the final result for the $\vec{E}_{l}$-odd imbalance induced by two transverse magnetic fields respectively odd and even:

$$
\begin{aligned}
\Delta_{L}^{(2)} D^{a t}\left(\vec{B}_{t}^{+}, \vec{B}_{t}^{-}\right) & =8 g_{F^{\prime}}^{2} \beta^{2} E_{l}^{2} \omega_{F^{\prime}}^{+} \omega_{F^{\prime}}^{-} \tau^{2} K \mathcal{F} \mathcal{S}_{c h i r}\left(\hat{\epsilon}_{X}, \hat{E}_{l}\right) \times \\
& \left(\left(\hat{B}_{t}^{+} \cdot \hat{E}_{l} \wedge \hat{\epsilon}_{e x}\right)\left(\hat{B}_{t}^{-} \cdot \hat{\epsilon}_{e x}\right)+\hat{B}_{t}^{-} \longleftrightarrow \hat{B}_{t}^{+}\right),
\end{aligned}
$$

where $\omega_{F^{\prime}}^{+}=\gamma B_{t}^{+}$, and $\omega_{F^{\prime}}^{-}=\gamma B_{t}^{-}$. After calibration:

$$
\theta_{\text {cal }} \frac{\Delta_{L}^{(2)} D^{a t}\left(\vec{B}_{t}^{+}, \vec{B}_{t}^{-}\right)}{D^{a t}\left(\theta_{c a l}\right)}=-\omega_{F^{\prime}}^{+} \omega_{F^{\prime}}^{-} \tau^{2}\left(\left(\hat{B}_{t}^{+} \cdot \hat{z} \wedge \hat{\epsilon}_{e x}\right)\left(\hat{B}_{t}^{-} \cdot \hat{\epsilon}_{e x}\right)+\hat{B}_{t}^{-} \longleftrightarrow \hat{B}_{t}^{+}\right) .
$$

When global rotations of the experiment are performed, the behavior of this signal is purely anisotropic.

\subsection{Generalization of the result to second-order magnetic pertur- bations of the pump and probe transitions}

Let us consider the perturbation of the probe transition by the transverse magnetic field $\vec{B}_{t}^{+}+\vec{B}_{t}^{-}$which is well known (see for instance [33]) to be responsible for a linear dichroism quadratic in this field, the so-called Voigt effect. Here we are interested in its $\vec{E}_{l}$-odd contribution $\propto B_{t}^{+} B_{t}^{-}$. In the evaluation of the second-rank tensor operator $\mathcal{O}_{i j}$ (Eq. (18)), the $\vec{B}_{t}$ field shows up in two different ways:

- it modifies the wavefunctions and thus the transition probabilities;

- it shifts the energy levels.

The magnitude of the perturbation is given by the ratio of the Zeeman frequency shift to the line width: $\omega_{F} / \Delta \omega \sim 10^{-5}$ per milligauss. In the present context, this effect can be considered as a second-order modification of the detection tensor (see Eq. 17) by the transverse magnetic field. We obtain this $\Delta^{(2)} \mathcal{D}_{i j}$ modification by performing the contraction of the two second-rank tensors, $\hat{B}_{j} \hat{B}_{k}$ which represents the perturbation and the unperturbed $\mathcal{D}_{i k}$ detection tensor:

$$
\Delta^{(2)} \mathcal{D}_{i j} \sim\left(\frac{\omega_{F^{\prime}}}{\Delta \omega}\right)^{2}\left(\hat{B}_{t}\right)_{i}\left(\hat{B}_{t}\right)_{k} \mathcal{D}_{j k} \sim\left(\frac{\omega_{F^{\prime}}}{\Delta \omega}\right)^{2}\left(\left(\hat{B}_{t}\right)_{i}\left(\hat{B}_{t} \cdot \hat{\epsilon}_{X}\right)\left(\hat{\epsilon}_{X}\right)_{j}-(X \leftrightarrow Y)\right) .
$$

When this detection tensor modification is contracted with the Stark alignment tensor, we get $\Delta^{(2)} D^{a t}\left(\vec{B}_{t}^{+}, \vec{B}_{t}^{-}\right)=\Delta^{(2)} \mathcal{D}_{i j} \cdot \mathcal{A}_{i j}^{S t}=\left(\frac{\omega_{F^{\prime}}}{\Delta \omega}\right)^{2}\left(\left(\hat{B}_{t} \cdot \hat{\epsilon}_{X}\right)\left(\hat{B}_{t} \cdot \hat{h}\right)\left(\hat{h} \cdot \hat{\epsilon}_{X}\right)-(X \leftrightarrow Y)\right)$ This yields a second-order correction to the atomic polarimeter imbalance:

$$
\theta_{\text {cal }} \frac{\Delta^{(2)} D^{a t}\left(\vec{B}_{t}^{+}, \vec{B}_{t}^{-}\right)}{D^{a t}\left(\theta_{c a l}\right)} \sim\left(\frac{\omega_{F^{\prime}}^{+} \omega_{F^{\prime}}^{-}}{\Delta \omega^{2}}\right)\left(\left(\hat{B}_{t}^{+} \cdot \hat{\epsilon}_{e x}\right)\left(\hat{B}_{t}^{-} \cdot \hat{z} \wedge \hat{\epsilon}_{e x}\right)+(+\leftrightarrow-)\right),
$$

where the expression into parentheses is actually identical to that appearing in Eq. (49). Note that the order of magnitude is also relatively close since $\Delta \omega \tau$ is not very different from unity.

The treatment given above can be applied without any difficulty to the second-order magnetic perturbation of the pump transition, leading to a very similar result. We conclude that the magnitude and the structure of the result is valid for any kind of second-order magnetic perturbation of the atomic system. 


\subsection{Combined effect of a misalignment and a magnetic perturbation}

As mentioned previously, it is possible to generate a $\vec{E}_{l}$-odd polarimeter imbalance by combining a magnetic perturbation and a pump-probe misalignment. This can be viewed as resulting from the contraction of the first-order perturbation, $\Delta_{m i s}^{(1)} \mathcal{D}_{i j}$ (Eq. (38) ) to the detector tensor and the first-order Larmor perturbation $\Delta_{L}^{(1)} \mathcal{A}_{i j}^{S t}(\tau)=-4 g_{F^{\prime}}^{2}\left(\left(\Delta_{L}^{(1)} \vec{b}_{S t}\right)_{i}\left(\vec{b}_{S t}\right)_{j}+(i \leftrightarrow j)\right)$ to the Stark alignment $\mathcal{A}_{i j}^{S t}(\tau)$ (Eq. (44)), we calculate the $\vec{E}_{l}$-odd atomic imbalance:

$$
\begin{aligned}
& \quad \Delta_{m i s L}^{(2)} D^{a t}=\Delta_{m i s}^{(1)} \mathcal{D}_{i j} \cdot \Delta_{L}^{(1)} \mathcal{A}_{i j}^{S t}(\tau) \\
& \quad=-8 g_{F^{\prime}}^{2} \beta^{2} E_{l}^{2} \omega_{F^{\prime}} \tau \delta \alpha K \mathcal{F} \mathcal{S}_{c h i r}\left(\hat{\epsilon}_{X}, \hat{E}_{l}\right)\left(\left(\hat{n} \cdot \hat{E}_{l} \wedge \hat{\epsilon}_{e x}\right)\left(\hat{B}_{t}^{-} \cdot \hat{\epsilon}_{e x}\right)+\left(\hat{B}_{t}^{-} \longleftrightarrow \hat{n}\right)\right),
\end{aligned}
$$

and the calibrated imbalance:

$$
\theta_{c a l} \frac{\Delta_{m i s L}^{(2)} D^{a t}\left(\vec{B}_{t}^{+}, \vec{B}_{t}^{-}\right)}{D^{a t}\left(\theta_{c a l}\right)}=\omega_{F^{\prime}} \tau \delta \alpha\left(\left(\hat{n} \cdot \hat{z} \wedge \hat{\epsilon}_{e x}\right)\left(\hat{B}_{t}^{-} \cdot \hat{\epsilon}_{e x}\right)+\left(\hat{B}_{t}^{-} \longleftrightarrow \hat{n}\right)\right) .
$$

We note again the expected correspondance between this result and that relative to Larmor precession treated to second order (Eq. (49)), when one performs the substitution:

$$
\omega_{F^{\prime}} \hat{B}_{t}^{+} \longrightarrow-\delta \alpha \hat{n} .
$$

\subsection{Absence of isotropic contribution. Anisotropy properties}

For all the effects considered in this section 5, the most important property already mentioned, is the absence of an isotropic contribution. In the quantities $\mathrm{S}_{\mathrm{xy}}=\frac{1}{2}\left(G_{x}+G_{y}\right)$ and $\mathrm{S}_{\mathrm{XY}}=\frac{1}{2}\left(G_{X}+G_{Y}\right)$, the spurious $\vec{B}_{t}^{+} \vec{B}_{t}^{-}$effect cancels out. Therefore, combining results obtained in two different input $\hat{\epsilon}_{e x}$ polarization configurations is sufficient to eliminate it as a source of systematics. However, some inconvenience remains as a result of the associated anisotropy.

If we introduce the angles $\theta_{B_{t}}^{+}=\left(\hat{y}_{0}, \vec{B}_{t}^{+}\right)$and $\theta_{B_{t}}^{-}=\left(\hat{y}_{0}, \vec{B}_{t}^{-}\right)$, this anisotropy is described by the angular dependence:

$$
\left(\sin \left(\phi-\theta_{B_{t}}^{-}\right) \cos \left(\phi-\theta_{B_{t}}^{+}\right)+\theta_{B_{t}}^{-} \longleftrightarrow \theta_{B_{t}}^{+}\right)=\sin \left(2 \phi-\left(\theta_{B_{t}}^{+}+\theta_{B_{t}}^{-}\right)\right) .
$$

This is similar to the anisotropy caused by the $\left(\vec{E}_{t} \cdot \vec{B}_{t}\right)^{+}$effect, but with different, and $a$ priori not simply related, anisotropy direction. Both effects can be present simultaneously.

This is why we cannot extract $\overrightarrow{G\left(\vec{E}_{t}, \vec{B}_{t}\right)}$ from the measured anisotropy. In order to obtain more information we shall have to rely on another property to be discussed in $\S 6.3$.

\section{Isotropy tests}

\subsection{Symmetry breaking effects and ways to reduce them}

We have listed in Table 1 the various candidates to systematic effects arising from cylindrical symmetry breaking defects. There are two main classes of such effects. Both have their magnitude characterized by a $2 \phi$-frequency modulation when the experiment is globally rotated. This implies that measurements in only four configurations obtained by successive rotations of $45^{\circ}$ are necessary to obtain full information. Effects of the first class are dangerous since 
Table 1: Summary of the various signals contributing to the polarimeter imbalance, with the amplitudes involved in the excitation process (1st column), the experimental defects involved (2nd column) and the angular dependence under global rotation of the experiment (3rd column). The symmetry-breaking defects are represented by a pair of dimensionless vector fields of small magnitude. From the product of the quantities appearing in columns 2 and 3 of each line, we obtain the atomic imbalance of the polarimeter (normalized by the calibration imbalance) which has to be compared with $\theta^{p v}$ to obtain the fractional importance of the systematic effect.

Origin

\section{APV Signal}

\section{Angular Dependence}

\section{Possible Systematics:}

interference

defects involved

$\beta E_{l} \beta E_{t}$

$\beta E_{l} \beta E_{t}$

$M_{1} \alpha E_{t}$

$\beta^{2} E_{l}^{2}$

$\beta^{2} E_{l}^{2}$

$\beta^{2} E_{l}^{2}$

$$
\begin{gathered}
\frac{E_{t}}{E_{l}} \hat{E}_{t}, \omega_{F} \tau \hat{B}_{t} \\
\frac{E_{t}}{E_{l}} \hat{E}_{t}, \delta \alpha \hat{n} \\
\frac{M_{1} \alpha E_{t}}{\beta^{2} E_{l}^{2}} \hat{E}_{t}, \frac{\gamma_{S} B_{t}}{\Delta W_{7 S}} \hat{B}_{t}
\end{gathered}
$$$$
\omega_{F^{\prime}}^{+} \tau \hat{B}_{t}^{+}, \omega_{F^{\prime}}^{-} \tau \hat{B}_{t}^{-}
$$$$
\omega_{F^{\prime}}^{-} \tau \hat{B}_{t}^{-}, \delta \alpha \hat{n}
$$$$
\frac{\omega_{F^{\prime}}^{+}}{\Delta \omega} \hat{B}_{t}^{+}, \frac{\omega_{F^{\prime}}^{-}}{\Delta \omega} \hat{B}_{t}^{-}
$$

\section{Class 1: Effects with isotropic contribution}

$$
\begin{gathered}
\left(\hat{E}_{t}^{+} \cdot \hat{B}_{t}^{+}-\left(\hat{B}_{t}^{+} \cdot \hat{\epsilon}_{e x}\right)\left(\hat{E}_{t}^{+} \cdot \hat{\epsilon}_{e x}\right)\right)+(+\longrightarrow-) \\
\left(\hat{E}_{t}^{+} \cdot \hat{n}-\left(\hat{n} \cdot \hat{\epsilon}_{e x}\right)\left(\hat{E}_{t}^{+} \cdot \hat{\epsilon}_{e x}\right)\right) \\
\left(\hat{B}_{t}^{+} \cdot \hat{\epsilon}_{e x}\right)\left(\hat{E}_{t}^{-} \cdot \hat{\epsilon}_{e x}\right)+(+\longleftrightarrow-)
\end{gathered}
$$

\section{Class 2: Effects without isotropic contribution}

$$
\begin{gathered}
-\left(\hat{B}_{t}^{+} \wedge \hat{z} \cdot \hat{\epsilon}_{e x}\right)\left(\hat{B}_{t}^{-} \cdot \hat{\epsilon}_{e x}\right)+(-\longleftrightarrow+) \\
\left(\hat{n} \wedge \hat{z} \cdot \hat{\epsilon}_{e x}\right)\left(\hat{B}_{t}^{-} \cdot \hat{\epsilon}_{e x}\right)+\left(\hat{B}_{t}^{-} \longleftrightarrow \hat{n}\right) \\
\left(\hat{B}_{t}^{+} \wedge \hat{z} \cdot \hat{\epsilon}_{e x}\right)\left(\hat{B}_{t}^{-} \cdot \hat{\epsilon}_{e x}\right)+(-\longleftrightarrow+)
\end{gathered}
$$

an isotropic contribution remains after our averaging the linear dichroism signal over those four configurations and represents a systematic effect superimposed on the APV signal. However, it is accompanied by an anisotropic contribution of the same order of magnitude which can indicate its presence. In order to reduce its magnitude one may proceed by reducing the spurious $\vec{E}_{t}$ and $\vec{B}_{t}$ fields as well as the pump-probe beam misalignment. Effects of the second class provide contributions which cancel out when averaged over the four input polarization configurations. They look harmless but in fact complicate the interpretation of the anisotropy, when present. This is why a correlation test is particularly welcome based on statistical data analysis which may establish absence of any significant link between the anisotropic and isotropic contributions (see § 6.2).

We can also conclude from this discussion how important it is to obtain as much information as possible about the stray fields and, even more so, to minimize them. We can determine the average value of the stray fields seen by the atoms simply by exploiting the physical effects analyzed in this work. By applying "large" $(\sim 2 \mathrm{G})$, controlled magnetic 
fields whose direction can be chosen and reversed at will, we can isolate the magnetoelectric dichroism described in section 4.1. From its magnitude, proportional to the stray $\vec{E}_{t}$ field and to the applied $\vec{B}_{t}$ field, we deduce the averaged magnitude and direction of the stray transverse electric field seen by the atoms inside the interaction region. We have found that the $\vec{E}_{l}$-even contribution is created by a density of electrons circulating inside the cell 23$]$. The field is radially distributed around the axis of the cylindrical cell. Since it cancels for optimal centring of the interaction region, we can reduce it. In addition, we can correct the $\vec{E}_{l}$-odd contribution by making tiny tilts (a few $\sim 10^{-3} \mathrm{rad}$ ) of the cell axis with respect to the common direction of the beams. In a similar way we learn about the transverse stray magnetic fields by deliberately amplifying the $\hat{B}_{t}^{+} \hat{B}_{t}^{-}$effects (class 2 effects, $\S 5.2$ ), using an applied field $\vec{B}_{t}$ of controlled direction. In order to disentangle the effects of classes 1 and 2 induced by the applied $\vec{B}_{t}$ field, we exploit their different behavior under global rotations of the experiment in addition to their different relative size when a different detection probe transition is used.

We note finally that optical rotation data are affected by the same stray fields which alter linear dichroism. We use this to obtain complementary information to monitor and reduce them.

\subsection{Statistical analysis of the data}

A complete set of data is obtained after cycling over four orientations of the input polarizations and provides us with one set of values of the four quantities $G_{y}, G_{Y}, G_{x}, G_{X}$, from which we extract $\mathrm{S}_{\mathrm{xy}}, \mathrm{S}_{\mathrm{XY}}$, the isotropic contributions and $\mathrm{D}_{x y}, \mathrm{D}_{X Y}$, the signal differences which may reveal anisotropic contributions (see Eqs. (35) - (37)). According to the previous considerations we can write:

$$
\mathrm{S}_{\mathrm{xy}}=\theta^{p v}+\mathrm{S}_{\mathrm{xy}}^{+}+\mathrm{S}_{\mathrm{xy}}^{-}
$$

where we recognize, besides the expected PV contribution, two effects of class 1 corresponding to $\vec{E}_{t}, \vec{B}_{t}$ fields of odd or even behaviour in $\vec{E}_{l}$ reversal (superscript + or - respectively). A similar equation holds also for $\mathrm{S}_{\mathrm{XY}}$. On another hand, the difference signal

$$
\mathrm{D}_{\mathrm{xy}}=\mathrm{D}_{\mathrm{xy}}^{+}+\mathrm{D}_{\mathrm{xy}}^{-}+\mathrm{D}_{\mathrm{xy}}^{(2)}
$$

also displays the contribution $\mathrm{D}_{\mathrm{xy}}^{(2)}$, resulting from effects of class 2 . It is interesting to underline the connection existing between $S_{x y}$ and $D_{x y}$ by reexpressing $D_{x y}$ as:

$$
\begin{aligned}
\mathrm{D}_{\mathrm{xy}} & =-\mathrm{S}_{\mathrm{xy}}^{+}\left(\cos 2 \theta_{E_{t}^{+}}-\cot \left(\theta_{B_{t}^{+}}-\theta_{E_{t}^{+}}\right) \sin 2 \theta_{E_{t}^{+}}\right)+(+\leftrightarrow-)+\mathrm{D}_{\mathrm{xy}}^{(2)} . \\
& =b^{+} \mathrm{S}_{\mathrm{xy}}^{+}+b^{-} \mathrm{S}_{\mathrm{xy}}^{-}+\mathrm{D}_{\mathrm{xy}}^{(2)} .
\end{aligned}
$$

The experimental data are to some extent noisy (mainly because of photon noise) and they constitute random variables. When a set of data is analyzed there are two questions to be answered: i) is the anisotropy statistically significant? ii) if so, is it possible to say whether there is an associated isotropic contribution?

i) First, in a cartesian coordinate system $\left(D_{x y}, D_{X Y}\right)$ we plot one point per data set. Figure 2 illustrates a cloud of 760 points. We look for any possible deviation of their gravity center with respect to the origin. For the data points of Fig. 2 this deviation is too small to be significant, it does not exceed one standard deviation. We conclude that this data set shows no significant anisotropy. 


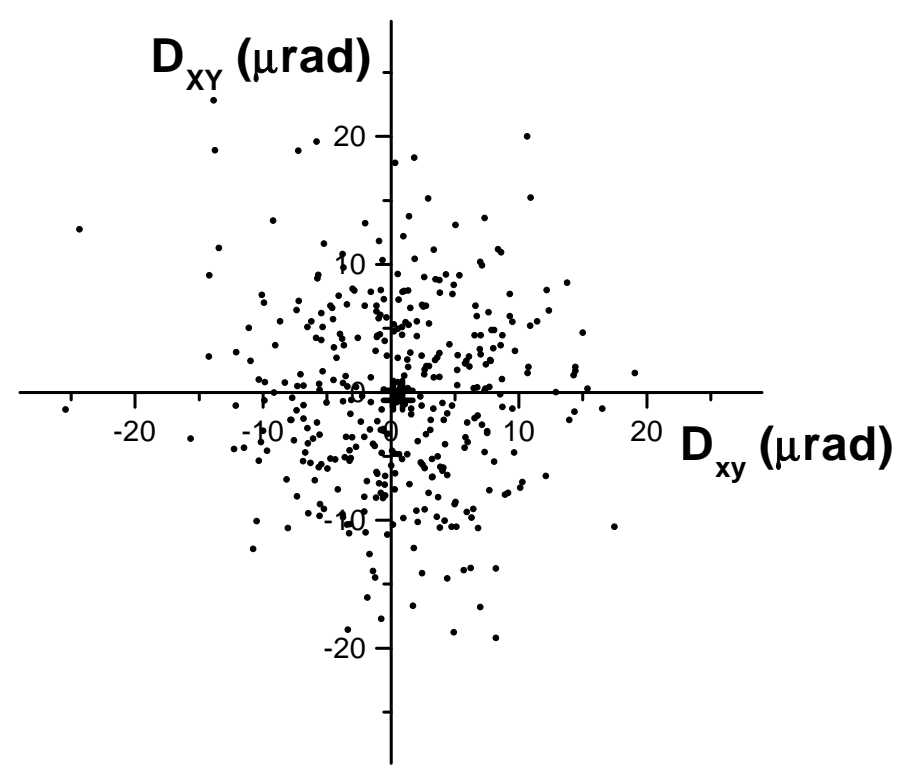

Figure 2: Anisotropy test performed on a sample of 760 sets of plane dichroism data (Eq. 26]) measured for the four different orientations of the input $\hat{\epsilon}_{e x}, \hat{\epsilon}_{p r}$ polarizations: for each individual set one signal difference, $D_{X Y}=G_{X}-G_{Y}$, is plotted versus the other one $D_{x y}=G_{x}-G_{y}$. On the cloud of points thus obtained, one looks for a distorsion with respect to a circular distribution centred on the origin. For the data presented here the center of gravity is indicated. Within the error bars, $\sigma_{D}=1.8 \times 10^{-7} \mathrm{rad}$, its coordinates merge into the origin.

ii) Second we evaluate the correlation coefficient $r$, between $S_{x y}$ and $D_{x y}$ (and similarly between $\mathrm{S}_{\mathrm{XY}}$ and $\mathrm{D}_{\mathrm{XY}}$ ).

$$
r=\frac{\left(\sum_{i=1}^{n} \mathrm{~S}_{\mathrm{xy}}{ }^{(i)} \mathrm{D}_{\mathrm{xy}}{ }^{(i)}\right) / n-<\mathrm{S}_{\mathrm{xy}}><\mathrm{D}_{\mathrm{xy}}>}{s_{\mathrm{S}} s_{\mathrm{D}}},
$$

where $<\mathrm{S}_{\mathrm{xy}}>$ and $\left\langle\mathrm{D}_{\mathrm{xy}}>\right.$ are the average values and $s_{\mathrm{S}}$ and $s_{\mathrm{D}}$ the standard deviations of $\mathrm{S}_{\mathrm{xy}}$ and $\mathrm{D}_{\mathrm{xy}}$ taken over the sample population. Thus, we can test the hypothesis that the population correlation is 0 against the alternative that it is not, with a chosen confidence level. According to Eq. (53), the existence of a linear relation between $\mathrm{D}_{\mathrm{xy}}$ and $\mathrm{S}_{\mathrm{xy}}$ indicates that, unless the class 2 effects are largely dominant or the class 1 effects absent, we should find a correlation between those two variables. According to a classical method of statistical analysis, see for instance [34, if the correlation turns out to be significant, we can obtain from the value of $r$ an estimation of the slope of the line of regression of $\mathrm{D}_{\mathrm{xy}}$ on $\mathrm{S}_{\mathrm{xy}}$, : $b=\frac{s_{\mathrm{D}}}{s_{\mathrm{S}}} r$, interpreted as $b^{+}$or $b^{-}$(see Eq. (53)).

In conclusion, the absence of correlation between the variables $D_{x y}$ and $S_{x y}$ (and between $\mathrm{D}_{\mathrm{XY}}$ and $\mathrm{S}_{\mathrm{XY}}$ ) is an important test. It enables us to conclude whether a non-zero average anisotropy is accompanied by an isotropic contribution which might alter the PV signal we want to detect. More precisely, the fraction of $\mathrm{D}_{\mathrm{xy}}$ which is correlated to $\mathrm{S}_{\mathrm{xy}}$, i.e. $b \mathrm{~S}_{\mathrm{xy}}$, yields an estimate of the systematic uncertainty which affects $<\mathrm{S}_{\mathrm{xy}}>$. 


\subsection{Order of magnitude of the residual systematic effect}

From the discussion presented in $\S 4$. and $\S 5$. it appears that the most worrying symmetry breaking effects come from the isotropic contribution of two stray fields $\vec{E}_{t}, \vec{B}_{t}$ or of a pumpprobe misalignment $\delta \alpha$ combined with an $\vec{E}_{t}^{+}$field. An estimate of the resulting systematic effect is directly derived from Eq. (34)

$$
G_{E B}=\left(\hat{z} \cdot \hat{E}_{l}\right) \frac{E_{t}}{E_{l}} \omega_{F^{\prime}} \tau,
$$

where $\omega_{F^{\prime}} \tau$ is the average Larmor precession angle of the angular momentum $\vec{F}^{\prime}$ during the time spent in the excited state, and from Eq. (39)

$$
G_{E \delta \alpha}=\left(\hat{z} \cdot \hat{E}_{l}\right) \frac{E_{t}}{E_{l}} \delta \alpha
$$

We record and average the harmful defects throughout the whole data acquisition by performing auxiliary measurements, at regular time intervals. If, over the whole averaging period, one achieves $\left\langle E_{t} / E_{l}\right\rangle \leq 0.5 \times 10^{-3}$ and $\left\langle B_{t}\right\rangle \leq 1 \mathrm{mG}$ leading to $\left\langle\omega_{F^{\prime}} \tau\right\rangle \leq$ $18 \mu \mathrm{rad}$, we arrive at $\left\langle G_{E B}>\leq 0.9 \times 10^{-2} \times \theta^{p v}\right.$, and it would make sense to go for one per cent statistically accurate measurements. Although keeping the defects reduced at this level is difficult to achieve, it does not look unfeasible. As to the geometrical pump-probe alignment, it is achieved using a reference four-quadrant photo-cell sensitive to both the pump and the probe beams (with respective radius 1.0 and $0.6 \mathrm{~mm}$ ); this cell is placed alternatively at the input and at the output of the oven, $30 \mathrm{~cm}$ apart. To ensure $\delta \alpha \leq$ $18 \times 10^{-6}$ rad requires a centering quality of $6 \mu \mathrm{m}$ on each quadrant cell. This seems achievable, since once achieved, the initial alignment is preserved during data averaging using servo-loops stabilizing both the pump and the probe beam positions on the initial reference, making use of auxiliary four-quadrant cells on pick up beams.

Finally, we mention a possible source of systematics which can be generated by the reflection of the pump beam on the output window of the cell: the reflected beam then goes back through the interaction region with a wave vector badly aligned with the probe wave vector. If a stray transverse, $\vec{E}_{l}$-even electric field is also present, this favors the systematic effect $G_{E \delta \alpha}$. This has prompted us to extinguish the reflexion on our cell windows by using temperature tuning of interferences taking place between the beams reflected by the inner and outer surfaces of each window 35.

\section{Conclusion}

To exploit the recent progress made using a new scheme for APV detection in cesium [11, we must suppress the systematic effects in order to match the statistical noise reduction expected in the next stage of our experiment aiming at a one percent precision. In the present paper, we have given a detailed discussion of a large class of systematic effects which all break the cylindrical symmetry of the ideal experimental configuration involved in the excitation and detection processes. A perfect experimental set-up would be invariant under both symmetries. The first is the mirror reflexion with respect to the plane defined by the linear polarization of the excitation beam and the electric field $\vec{E}_{l}$, colinear to this beam; a breaking of this is evidence for APV. The second is the cylindrical symmetry around the common direction of the pump and probe beams. In an ideal design, the PV signal would be invariant under any global rotation of the experiment around this direction. 
Experimental defects which break mirror symmetry do not necessarily break the cylindrical invariance: this is the case with a longitudinal magnetic field, which happens to be odd under $\vec{E}_{l}$ reversal. The effect of such a field has been considered previously [23] and we merely recall here that an auxiliary Faraday effect measured on a different probe line allows us to correct for it.

In the present work, our aim was to find solutions to the broader problem arising from the experimental defects, namely stray fields and misalignments which break both mirror and cylindrical symmetry properties. We began by a short review of the properties of the chiral optical gain, which gives rise to the PV signal in our experiment. This originates from a mirror symmetry breaking contribution to the excited state atomic alignment, which reflects the presence of a PV transition amplitude in the excitation process, resulting from the weak PV electron-nucleus interaction.

On this occasion, we have found it convenient to introduce a tensor formalism for calculating the atomic alignment in the excited state and the detection signal. This allows us to incorporate in a systematic way the contributions arising from all the $6 \mathrm{~S}-7 \mathrm{~S}$ transition amplitudes, in presence of transverse $\vec{E}_{t}$ and $\vec{B}_{t}$ fields breaking the cylindrical symmetry of the experiment. Our two-channel polarimetry measurements performed in balanced mode provide us with a differential, pseudoscalar signal which makes possible dark field detection of the PV effect in the ideal configuration. We have derived a general expression for the differential polarimeter imbalance, this results from the contraction of two second-rank tensors, the alignment tensor and a detection tensor constructed from the eigenpolarizations of the polarimeter.

It is remarkable that both the APV and the calibration signals are invariant under simultaneous rotations of the input pump and probe polarizations around the direction common to both beams. During such rotations, stray transverse fields and misalignment remain fixed so that their relative direction with respect to the beam polarizations are modified. As a result they generate new contributions to the atomic polarimeter imbalance. We have endeavored to answer two crucial questions:

- are these contributions distinguishable from the true PV signal?

- can we extract enough information from their variations observed during the rotation of the experiment to separate the true PV signal from systematic effects?

Our analysis shows that associated with each defect there is either a new contribution to the atomic alignment tensor in the excited state or a tiny rotation of this tensor or (else) of the detection tensor. The tensor formalism introduced at the outset of the paper is well adapted to derive all the corrections to the imbalance to second order in the defects. In order for there to be an $\vec{E}_{l}$-odd imbalance which simulates the PV signal at least two defects must conspire. The possible pairs can be arranged into two classes. Both have their magnitude characterized by a $\cos (2 \phi)$ modulation as the rotations are performed through $180^{\circ}$. After we average the results over four configurations obtained by successive rotations of $45^{\circ}$, a systematic effect may only result from the effects belonging to the first class, which involves the presence of a transverse electric field. To reduce the average transverse $\vec{E}_{t}$ field seen by the atoms, we perform auxiliary sequences of measurements with a known applied magnetic field. We must also compensate carefully the transverse magnetic fields, using the atomic signals described in this work.

After optimization, there is the possibility of a left-over anisotropic contribution which could be associated both with class 1 and class 2 processes. We have found a way to get information about the harmful class 1 isotropic contribution by showing that its fluctuations are correlated with those of the class 1 anisotropy contribution. ( No such correlation of 
course can exist for the APV purely isotropic term). A statistical treatment of the data yields an estimate of the systematic uncertainty associated with the class 1 processes.

As an aside remark, we would like to point out that, during the course of this work, we have established a connection between the most worrying sources of systematics, generated by parallel components of transverse electric and magnetic fields, and the magnetoelectric Jones dichroism [30 requiring extreme conditions for being observed in liquid samples [31. This highlights the extreme sensitivity of highly forbidden transtions, such as the cesium $6 S-7 S$, to the symmetry of the experimental set-up and illustrates the great variety of new processes which can be studied both theoretically and experimentally.

\section{Acknowledgements}

We are very grateful to Claude Bouchiat for many fruitul discussions and to Mark Plimmer for critical reading of the manuscript.

\section{References}

[1] M. A. Bouchiat and C. Bouchiat, J. Phys. France 35, 899 (1974) and Rep. Prog. Phys. 60, 1351 (1997).

[2] M. J. D. Macpherson et al., Phys. Rev. Lett. 67, 2784 (1991).

[3] D. M. Meekhof et al., Phys. Rev. Lett. 71, 3442 (1993).

[4] J. N. H. Edwards et al., Phys. Rev. Lett. 74, 2654 (1995); P. Vetter et al., Phys. Rev. Lett. 74, 2658 (1995).

[5] M. A. Bouchiat, J. Guéna, L. Hunter and L. Pottier, Phys. Lett. B 117, 358 (1982); ibid B 134, 463 (1984); J. Phys. (France) 47, 1709 (1986).

[6] P. S. Drell and E. D. Commins, Phys. Rev. A 32, 2196 (1985).

[7] S. L. Gilbert and C.E. Wieman, Phys. Rev. A 34, 792 (1986).

[8] C. S. Wood, et al., Science 275, 1759 (1997).

[9] S. C. Bennett and C. E. Wieman, Phys. Rev. Lett. 82, 2484 (1999).

[10] C.S. Wood et al., Can. J. Phys. 77, 7 (1999); B.P. Masterson et al., Phys. Rev. A 47, 2139 (1993).

[11] J. Guéna et al., e-print physics/0210069 Phys. Rev. Lett. 90, 143001 (2003)

[12] D. Chauvat, et al., Eur. Phys. J. D 1, 169 (1998).

[13] J. Guéna et al., Quantum Semiclass. Opt. 10, 733 (1998).

[14] M. A. Bouchiat and C. Bouchiat, J. Phys. France 36, 493 (1975).

[15] V. A. Dzuba, V. V. Flambaum and O. P. Sushkov, Phys. Lett. A 141, 147 (1989); S. A. Blundell, J. Sapirstein and W. R. Johnson, Phys. Rev. D 45, 1602 (1992).

[16] A. Derevianko, Phys. Rev. Lett. 85, 1618 (2000). 
[17] A.I. Milstein, O.P. Sushkov, I. S. Terekhov e-print hep-ph/0109257 Phys. Rev. Lett. 89, 283003 (2002); M. Yu. Kuchiev and V. V. Flambaum, Phys. Rev. Lett. 89, 283002 (2002).

[18] V. A. Dzuba et al., Phys. Rev. D 66, 076013 (2002).

[19] M. A. Bouchiat, Ph. Jacquier, M. Lintz, L. Pottier, Opt.Commun. 56, 100 (1985).

[20] M. A. Bouchiat, J. Guéna, Ph. Jacquier, M. Lintz and L. Pottier, J. Phys. France 50, 157 (1989).

[21] M. A. Bouchiat and C. Bouchiat, Z. Phys. D 36, 105 (1996).

[22] J. Guéna et al., J. Opt. Soc. Am. B 14, 271 (1997) and Opt. Commmun. 119, 403 (1995).

[23] J. Guéna et al., Appl. Phys. B 75, 739 (2002).

[24] M. A. Bouchiat et al., Z. Phys. D 33, 89 (1995).

[25] D. Chauvat et al., Opt. Commmun. 138, 249 (1997).

[26] S. Ciraci and Inder P. Batra, Phys. Rev. B 28, 982 (1983).

[27] J.A. Rodriguez et al., J. Phys. Chem. 100, 18240 (1996).

[28] M. Lintz and M. A. Bouchiat, Surf. Sci. 511, L319 (2002).

[29] K. R. Zavadil, J. L. Ing, Conference 950110 American Institute of Physics (1995); M. Brause, et al., Surf. Sci. 383, 216 (1997).

[30] R. C. Jones, J. Opt. Soc. Am. 38, 671 (1948).

[31] T. Roth and G. L. J. A. Rikken, Phys. Rev. Lett. 85, 4478 (2001).

[32] D. Budker and J. E. Stalnaker, physics/0302096

[33] For a review article of resonant magneto-optical effects in atoms see: D. Budker, W. Gawlik, D.F. Kimball, S.M. Rochester, V.V. Yashchuk, A. Weis, Rev. Mod. Phys. 74, 1153 (2002), §VI.

[34] L. L. Chao, in Statistics Methods and Analyses , (Mc-Graw-Hill, 2nd edition, 1974), ch. 14 .

[35] E. Jahier et al., Appl. Phys. B 71 , 561 (2000). 